ARTICLE

https://doi.org/10.1038/s41467-019-10954-y

\title{
Cell type-dependent differential activation of ERK by oncogenic KRAS in colon cancer and intestinal epithelium
}

\author{
Raphael Brandt (i) 1,9, Thomas Sell (10 1,2,9, Mareen Lüthen (1) 1,3, Florian Uhlitz ${ }^{1,2,3}$, Bertram Klinger ${ }^{1,2}$, \\ Pamela Riemer (10) ${ }^{1}$, Claudia Giesecke-Thiel (1) 4,5, Silvia Schulze ${ }^{1}$, Ismail Amr El-Shimy (1) 1,2, Desiree Kunkel ${ }^{6}$, \\ Beatrix Fauler ${ }^{5}$, Thorsten Mielke (1) ${ }^{5}$, Norbert Mages ${ }^{5}$, Bernhard G. Herrmann (1) ${ }^{5,7}$, Christine Sers (1) 1,3,8, \\ Nils Blüthgen (10) $1,2,3,8,10$ \& Markus Morkel (1) 1,3,8,10
}

Oncogenic mutations in KRAS or BRAF are frequent in colorectal cancer and activate the ERK kinase. Here, we find graded ERK phosphorylation correlating with cell differentiation in patient-derived colorectal cancer organoids with and without KRAS mutations. Using reporters, single cell transcriptomics and mass cytometry, we observe cell type-specific phosphorylation of ERK in response to transgenic KRASG12V in mouse intestinal organoids, while transgenic BRAF ${ }^{\mathrm{V} 600 \mathrm{E}}$ activates ERK in all cells. Quantitative network modelling from perturbation data reveals that activation of ERK is shaped by cell type-specific MEK to ERK feed forward and negative feedback signalling. We identify dual-specificity phosphatases as candidate modulators of ERK in the intestine. Furthermore, we find that oncogenic KRAS, together with $\beta$-Catenin, favours expansion of crypt cells with high ERK activity. Our experiments highlight key differences between oncogenic BRAF and KRAS in colorectal cancer and find unexpected heterogeneity in a signalling pathway with fundamental relevance for cancer therapy.

\footnotetext{
${ }^{1}$ Institute of Pathology, Charité Universitätsmedizin Berlin, Charitéplatz 1, 10117 Berlin, Germany. ${ }^{2}$ IRI Life Sciences, Humboldt University Berlin, Philippstrasse 13, 10115 Berlin, Germany. ${ }^{3}$ German Cancer Consortium (DKTK), German Cancer Research Center (DKFZ), 69120 Heidelberg, Germany. ${ }^{4}$ Department of Cell Biology, German Rheumatism Research Center, Leibniz Institute, Berlin, Germany. ${ }^{5}$ Max Planck Institute for Molecular Genetics, Ihnestr. 73, 14195 Berlin, Germany. ${ }^{6}$ Berlin-Brandenburg Center for Regenerative Therapies (BCRT), Charité - Universitätsmedizin Berlin, Campus Virchow-Klinikum, Augustenburger Platz 1, 13353 Berlin, Germany. ${ }^{7}$ Institute for Medical Genetics, Charité Universitätsmedizin Berlin, Hindenburgdamm 30, 12203 Berlin, Germany. ${ }^{8}$ Berlin Institute of Health (BIH), Anna-Louise-Karsch-Str. 2, 10178 Berlin, Germany. ${ }^{9}$ These authors contributed equally: Raphael Brandt, Thomas Sell. ${ }^{10}$ These authors jointly supervised this work: Nils Blüthgen, Markus Morkel. Correspondence and requests for materials should be addressed to N.B. (email: nils.bluethgen@charite.de) or to M.M. (email: markus.morkel@charite.de)
} 
M ultiple signalling pathways, including the mitogenactivated protein kinase (MAPK) and $\mathrm{Wnt} / \beta$-catenin cascades, form a network controlling cellular turnover in the intestinal epithelium ${ }^{1}$. Collectively, activities within the signalling network control stem cell maintenance, cell proliferation, differentiation into absorptive enterocyte and secretory cells, and apoptosis. Wnt $/ \beta$-catenin and MAPK activities are regionalised within the folded single-layered intestinal epithelium. Both are high in crypts harbouring stem cells and low in differentiated cells that have migrated away from the crypt base. Oncogenic mutations activating $\beta$-catenin and MAPK perturb intestinal homeostasis and thereby drive colorectal cancer (CRC) initiation and progression.

MAPK modules transduce signals downstream of receptor tyrosine kinases and RAS family GTPases. The consecutive RAF, MEK and ERK kinases represent a MAPK module frequently activated in cancer. ERK can phosphorylate and activate a series of transcription factors orchestrating a complex cellular response that often is pro-proliferative ${ }^{2}$. In the normal intestine, EGFR to ERK signalling is initiated by ligands from the crypt microenvironment, which are secreted by e.g. epithelial Paneth cells of the small intestine, Reg4+ secretory niche cells of the large intestine, or adjacent fibroblasts ${ }^{3,4}$. In CRC, ERK activity is supposedly more cell-autonomous due to oncogenic mutations activating KRAS, NRAS or BRAF (found in 45,5 and $10 \%$ of CRCs, respectively) ${ }^{5,6}$, or by de novo expression of EGFR ligands such as amphiregulin ${ }^{7}$. Signal transduction to ERK is a main determinant of cancer development and therapy response $e^{5,6,8}$.

Recent studies suggest that the relationship between ERKactivating mutations, ERK activity and phenotypic outcome in CRC is complex. Firstly, mutations in KRAS and BRAF are associated with distinct CRC development routes: KRAS, but not BRAF, mutations frequently occur as secondary events after mutations activating $\mathrm{Wnt} / \beta$-catenin in the conventional CRC progression sequence ${ }^{9,10}$. Conversely, BRAF, and less frequently KRAS, mutations precede activation of $\mathrm{Wnt} / \beta$-catenin in the alternative serrated progression route ${ }^{11,12}$. The observed disequilibrium between KRAS and BRAF mutations in the conventional vs. serrated pathways of CRC evolution suggest the existence of functional differences, resulting in distinctive effects on clinical course and treatment efficacy ${ }^{13}$. Secondly, ERK activity appears to be heterogeneous in genetically identical CRC cells. Cells at the invasive front frequently exhibited higher ERK phosphorylation levels compared with cells in central areas of the same cancer, and CRCs with activating KRAS mutations also showed heterogeneous ERK activity ${ }^{14}$. Previous studies already showed heterogeneous $\mathrm{Wnt} / \beta$-catenin activity in cancer specimens, suggesting a more general paradigm of graded pathway activities in $\mathrm{CRC}^{15}$. Furthermore, CRC cells have been shown to exhibit functional differences within a cancer, as only few CRC cells, so-called cancer stem cells, could initiate new tumours in xenografts $^{16-19}$. Gradients of surface markers, such as EphB2, were found to distinguish CRC stem cells with high malignant potential ${ }^{20}$. CRC subtypes can share similarities with cell types of the normal crypt, such as stem cells, enterocytes or secretory cells in bulk cell analysis ${ }^{21}$. Finally, because of variable signal transduction and differentiation states, genetically identical CRC clones exhibit variable proliferative potential and therapeutic response $^{22}$.

Experimental techniques with cellular resolution, ranging from fluorescent reporters ${ }^{23}$ to single-cell transcriptome analyses ${ }^{24,25}$ and mass cytometry ${ }^{26}$ hold the promise to disentangle the relationship between oncogenes, cell differentiation states and cellsignal transduction while taking into account cellular heterogeneity. Here, we ask whether oncogenic forms of KRAS or BRAF show cell-to-cell heterogeneity in their proclivity to activate ERK.
For this, we use patient-derived and mouse transgenic organoid cultures that maintain the cell hierarchy of tissue in vitro ${ }^{27}$. We assess signalling network states with cellular resolution by mass cytometry and use BRAFV600E and KRAS ${ }^{\mathrm{G} 12 \mathrm{~V}}$ transgenes to assess immediate impact of the oncogenes on cell-signal transduction, gene expression programmes and phenotypic outcome. We discover strong functional differences between the BRAF and KRAS oncogenes and find that signal transduction from KRAS to ERK is shaped by different strengths of feed forward and negative feedback in a cell type-specific manner.

\section{Results}

Graded ERK activity in KRAS-mutant CRC organoids. To investigate whether oncogenic KRAS enforces constitutive activity of MEK and ERK kinases, we examined patient-derived threedimensional CRC organoid cultures by immunohistochemistry. We found heterogeneous phosphorylation of both, MEK and ERK, in organoids with no mutations in the EGFR-RAS-ERK cascade (line OT326), as well as in KRAS-mutant organoids (line OT227, carrying a KRAS ${ }^{\mathrm{G} 13 \mathrm{D}}$ mutation) (Fig. 1a).

We next used mass cytometry to analyse cell differentiation markers and MEK and ERK phosphorylation side-by side in the patient-derived organoids (Fig. 1b). We selected the two organoid lines used above, as well as line OT302, harbouring a KRASG12D mutation. We found that cells of all three organoid lines formed gradients with respect to levels of EphB2, a known marker of CRC hierarchies linked to metastasis and therapy response $\mathrm{e}^{20}$. In two of the three lines (OT326 and OT302), a substantial proportion of EphB2-low cells was marked by cleaved Caspase 3 , suggesting apoptotic removal of cells at the end of their life span. Intriguingly, all three lines displayed gradients of phosphorylated MEK and ERK that were largely congruent with EphB2. These results indicate that patient-derived CRC organoids contain phosphorylation gradients of MEK and ERK kinases along an axis defined by cell differentiation. The observed gradient formed regardless of oncogenic activation of the upstream KRAS GTPase, and in the absence of tumour stroma that is not present in the organoids.

BRAFV600E, but not KRAS $^{\mathrm{G} 12 \mathrm{~V}}$, induces strong ERK activity. As MEK and ERK activities were graded along a differentiation axis in patient-derived CRC organoids irrespective of mutational status of KRAS, we asked whether oncoproteins activating the MEK-ERK signalling axis exert their activities in a cell typespecific manner. To study this question, we employed intestinal organoids of transgenic mice carrying doxycycline-inducible single copy constructs encoding tdTomato linked to KRASG12V, BRAF $^{\mathrm{V} 600 \mathrm{E}}$, or firefly luciferase (FLUC) as a control in the Gt (ROSA26)Sor locus (Fig. 2a) 28,29 .

We initiated organoid cultures by embedding intestinal crypts from FLUC-, KRAS $\mathrm{K}^{2} \mathrm{~V}_{-}$, and $\mathrm{BRAF}^{\mathrm{V} 600 \mathrm{E}_{\text {-inducible mice into }}}$ extracellular matrix, as described before ${ }^{27}$. When we induced oncoprotein production by adding doxycycline to the culture media, $\mathrm{BRAF}^{\mathrm{V} 600 \mathrm{E}}$ led to irreversible disintegration of organoids within 1-2 days, whereas transgenic KRASG12V or the FLUC control protein were well tolerated, even after several passages (Fig. 2b). To examine whether the BRAF oncogene has detrimental effects on the epithelium beyond the previously reported loss of stem cells ${ }^{29,30}$, we examined histology of the induced organoids at ultrastructural level using transmission electron microscopy (Fig. 2c). We found that control and KRASG12V -induced organoids showed the expected tissue structure, that is, a single-layered polarised epithelium with continuous apical and basal surfaces as well as a brush border at the apical side. Desmosomes, providing lateral cell adhesion, were 
a
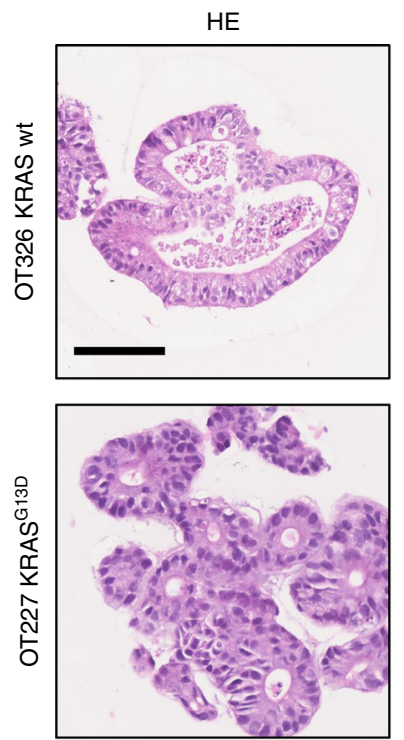

b
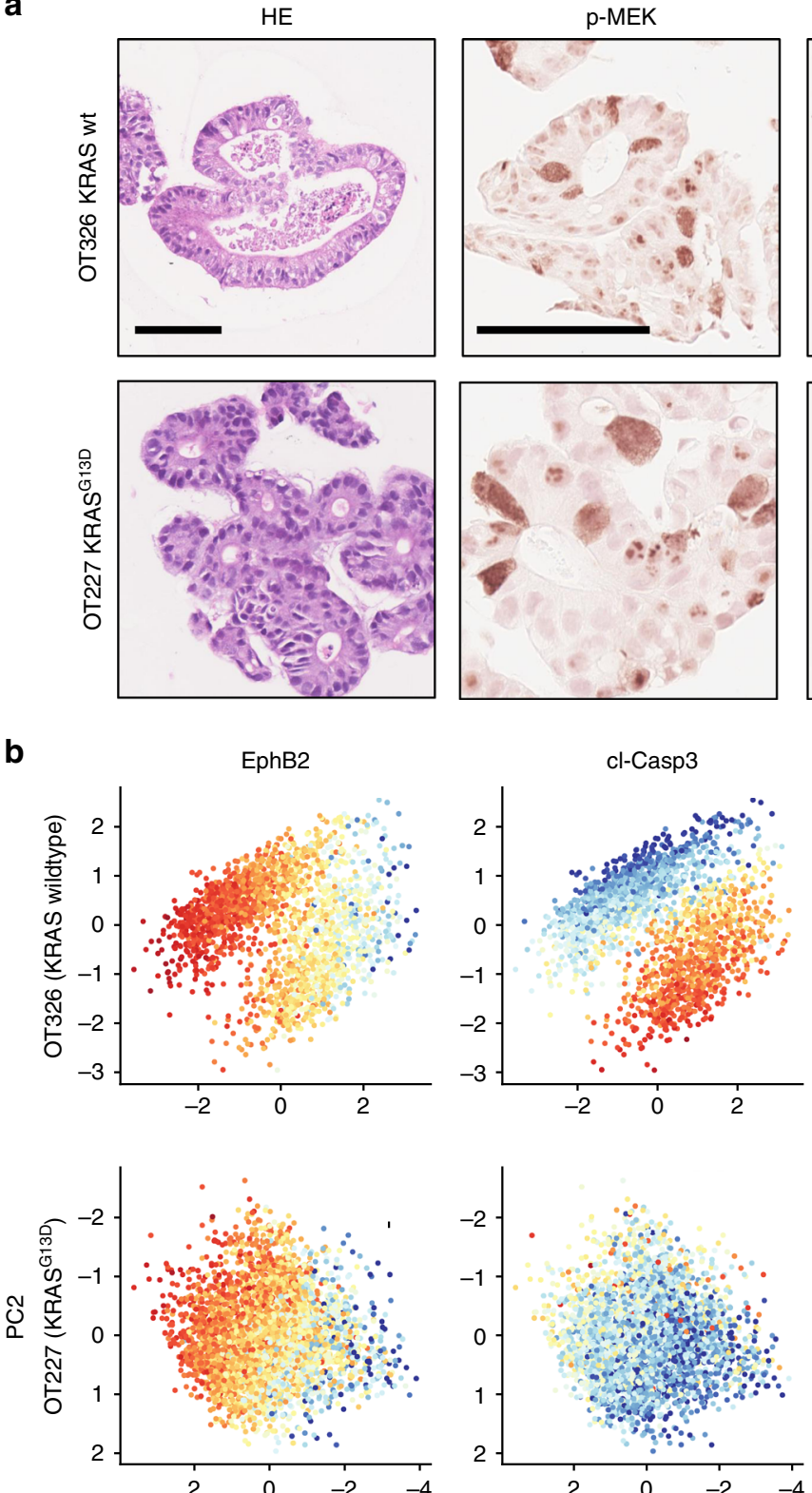

cl-Casp3
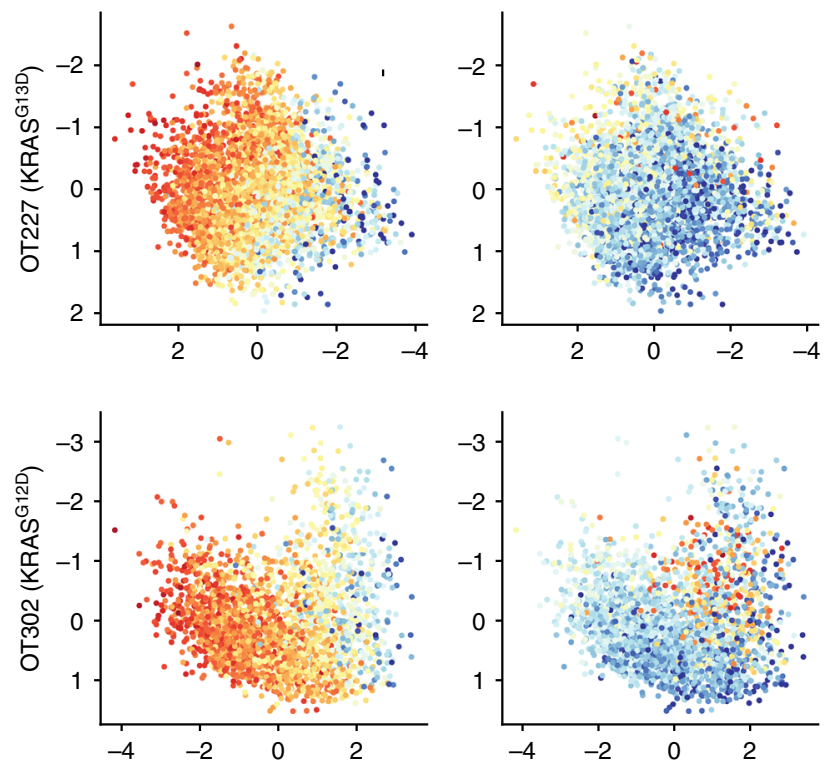

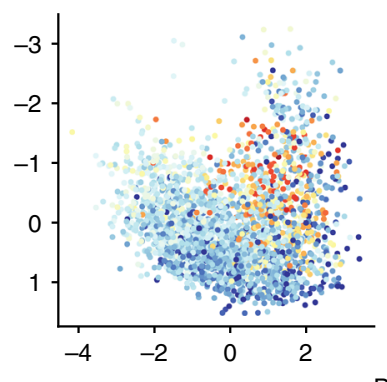

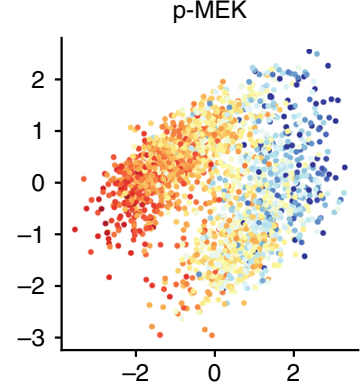
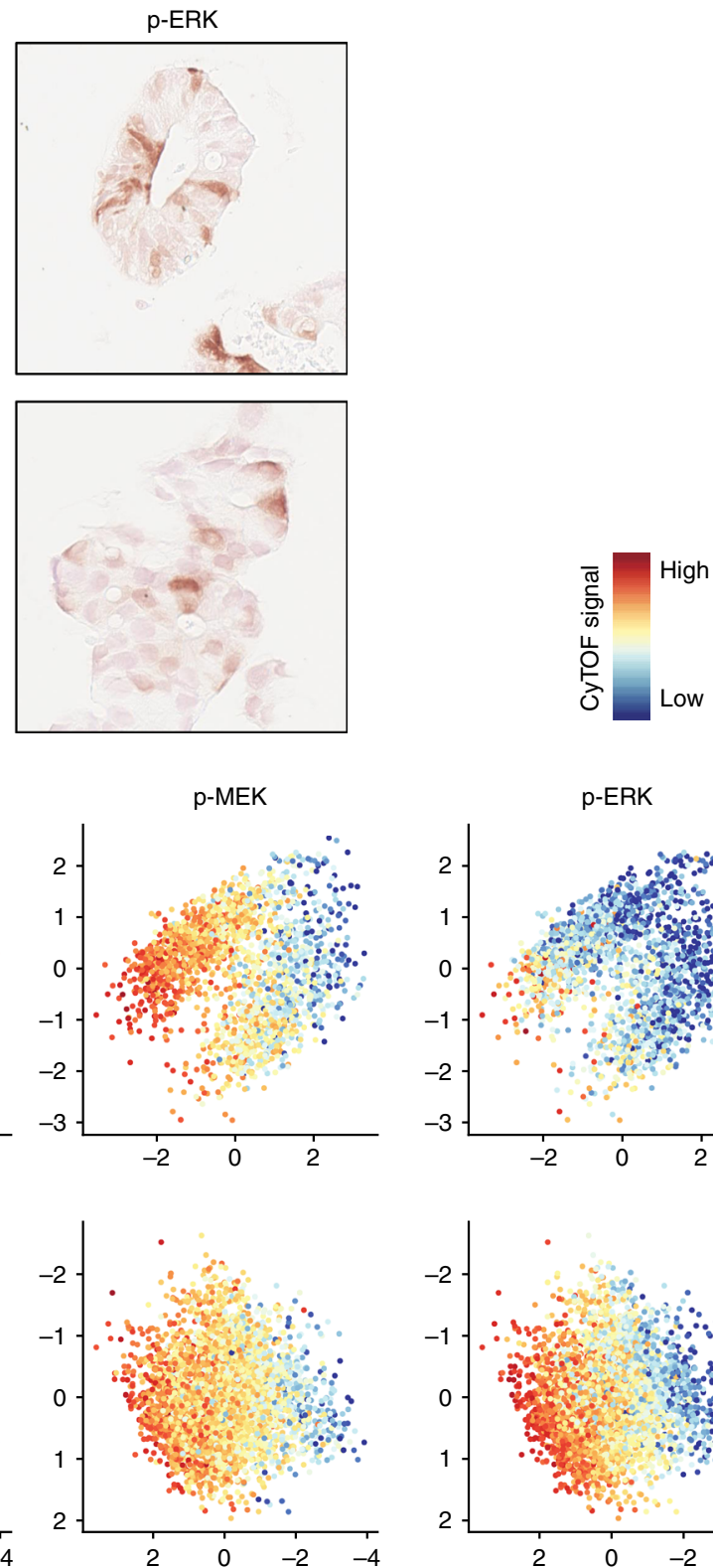

p-ERK
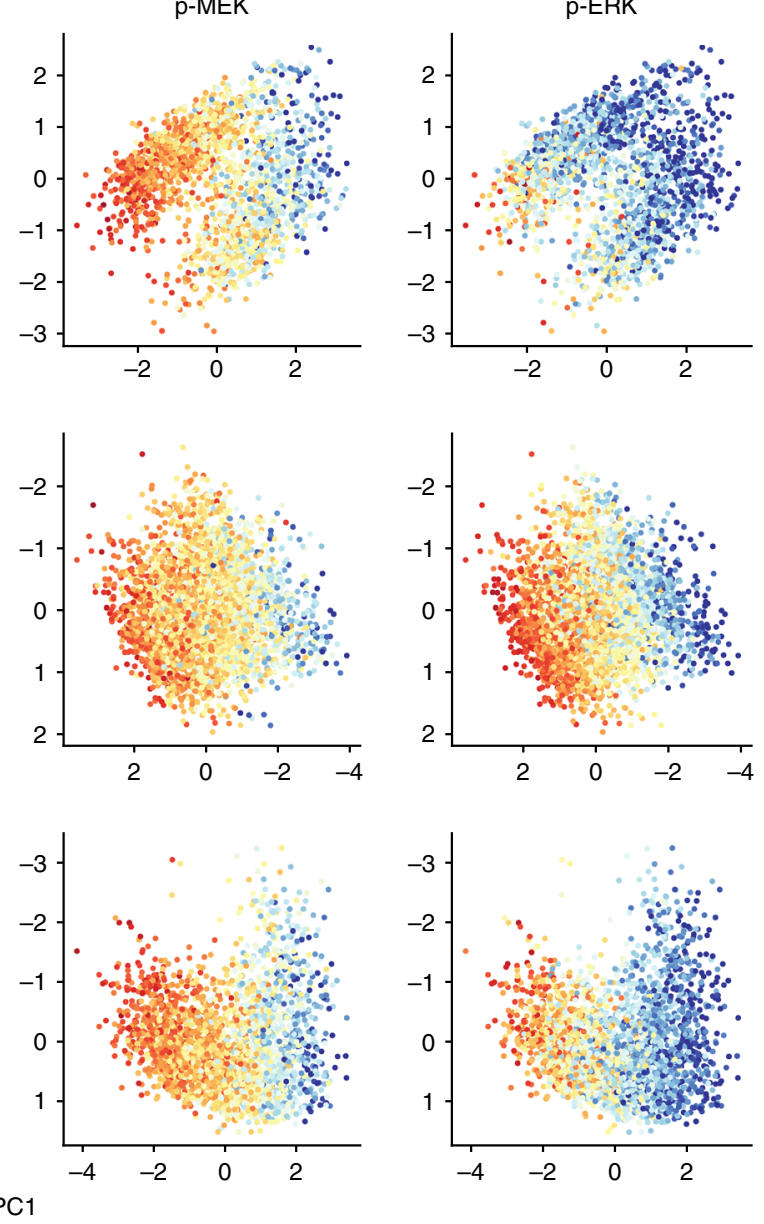

Fig. 1 Graded MEK and ERK phosphorylation in patient-derived organoids. a Haematoxilin-eosin (HE) staining and phospho-MEK and phospho-ERK immunohistochemistry of two PD3D lines OT326 and OT227 that are KRAS-wild-type and KRAS-mutant, respectively. Scale bars denote $100 \mu \mathrm{m}$ for HE and immunohistochemistry. b CyTOF analysis of PD3Ds. Principal component analyses, colour-coded for EphB2, cleaved Caspase, phospho-MEK and phospho-ERK are shown. Red, yellow and blue colours of the scale represent high, intermediate and low signals, respectively. CyTOF data is available as a Source Data file 
a

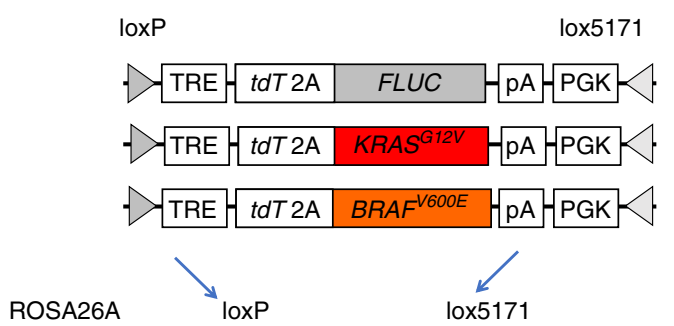

b
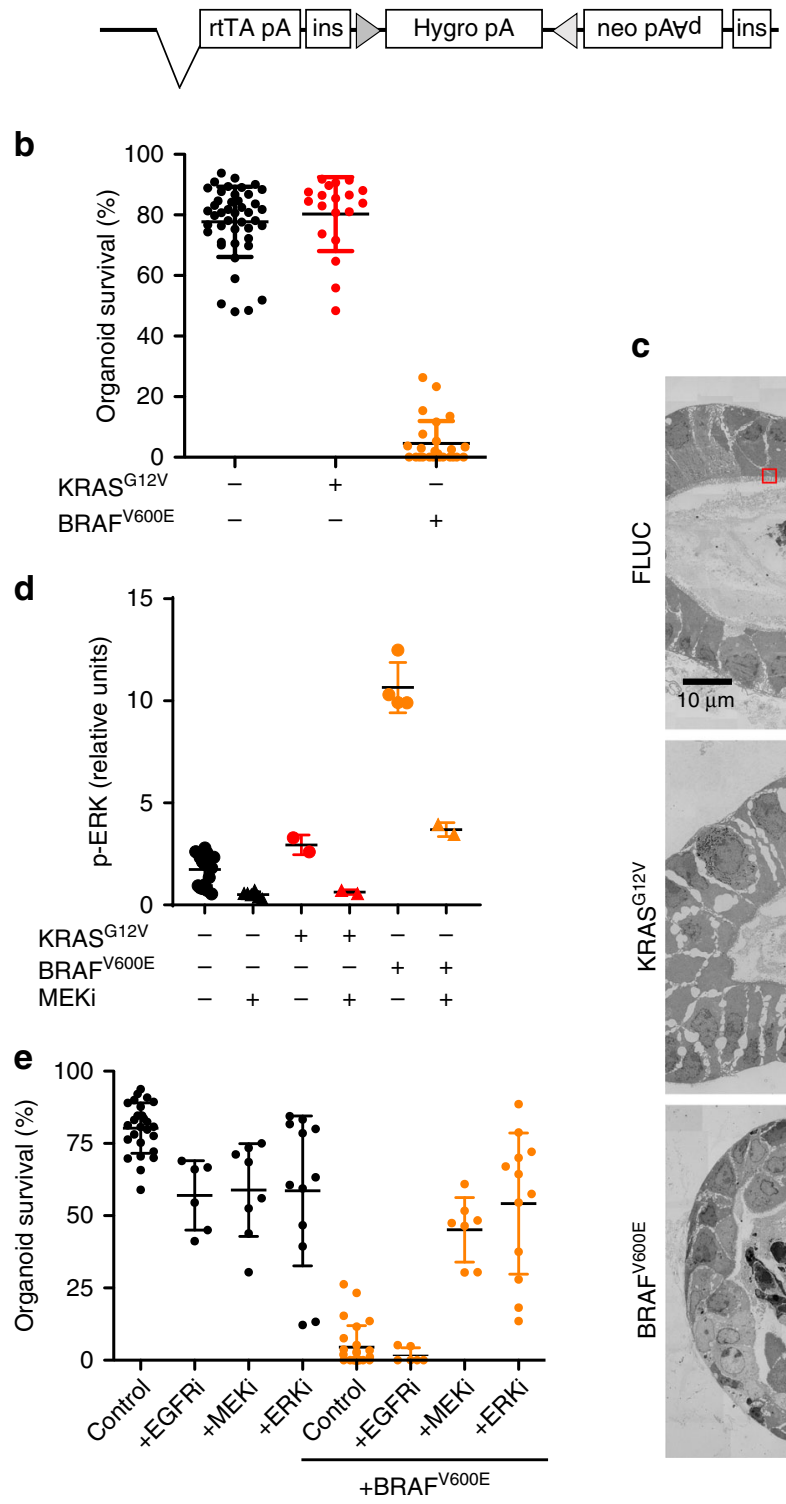

C
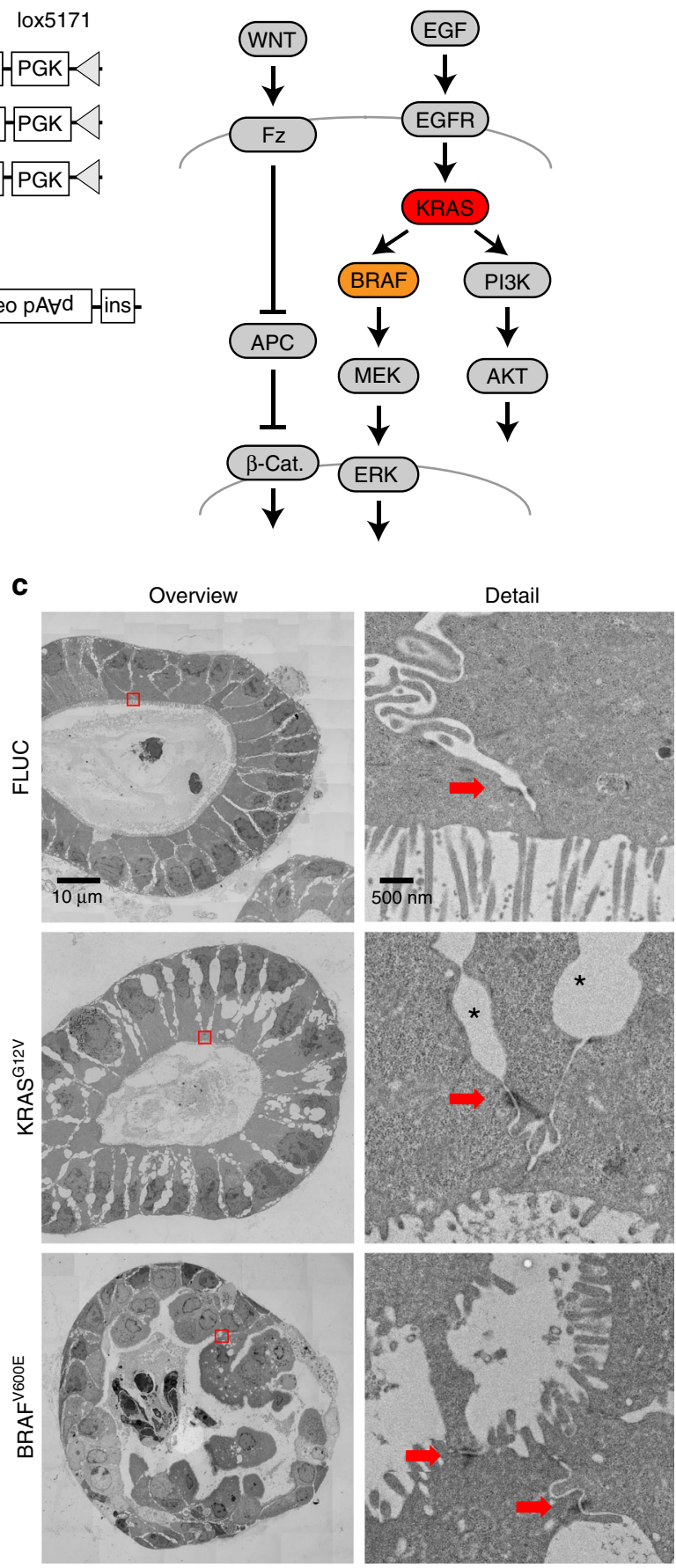

Fig. 2 Transgenic BRAFV600E, but not KRASG12V disrupts organoids due to high ERK activity. a Simplified representations of transgenes and the RAS-ERK and $\mathrm{Wnt} / \beta$-catenin pathways, indicating relative positions of the KRAS and BRAF proto-oncogenes. $\mathbf{b}$ Organoid survival 4 days after induction of oncogenic $\mathrm{KRAS}^{\mathrm{G} 12 \mathrm{~V}}$ or BRAF $\mathrm{V} 600 \mathrm{E}$. Organoids are counted immediately after passaging, and fractions of surviving organoids were calculated at day 4. Control organoids comprise of mixed non-induced cultures of KRASG12V and BRAFV600E lines. c Electron microscopy reveals loss of epithelial integrity after BRAFV600E induction. Images of the intestinal organoid epithelium, $24 \mathrm{~h}$ after induction of control FLUC, KRASG12V or BRAFV600E transgenes. Detailed views (right) represent a zoom into areas marked by red boxes in the overviews (left). Detailed views show apical surfaces of adjacent enterocytes with brush border. Red arrows mark desmosomes. Intercellular vacuoles, most visible in the KRASG12V model (marked by *) are likely fixation-induced artefacts, see ref. ${ }^{27}$. Scale bars are $10 \mu \mathrm{m}$ in the overview panels and $500 \mathrm{~nm}$ in the detailed view panels. $\mathbf{d}$ Quantification of ERK phosphorylation in organoids, $24 \mathrm{~h}$ after induction of control, BRAF or KRAS transgenes, using a capillary protein analysis. e Quantification of organoid survival, 4 days after inhibition of EGFR, MEK, ERK and/or induction of BRAFV600E, as in panel (b). Error bars in panels (b), (d) and (e) denote standard deviations. Data shown in panels (b), (d), and (e) are available as a Source Data file 
clearly visible. In contrast, $\mathrm{BRAF}^{\mathrm{V} 600 \mathrm{E}}$-induced organoids displayed a continuous basal surface, whereas the apical side was grossly distorted, although it contained a brush border as evidence of polarisation. Nuclei were pleomorphic and no longer lined up basally but scattered at different positions. Cells were still attached to each other by desmosome bridges, indicating that the ongoing epithelial disorganisation was taking place in the presence of lateral cell adhesion.

To ascertain whether the epithelial disorganisation provoked by $\mathrm{BRAF}^{\mathrm{V} 600 \mathrm{E}}$ was correlated with MAPK activity, we measured phosphorylation of ERK. We found that induction of BRAF ${ }^{V 600 E}$, but not KRASG12V, resulted in high phospho-ERK levels in intestinal organoids, as determined by capillary protein analysis

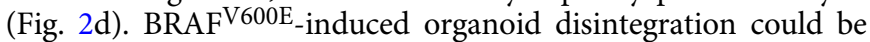
counteracted by inhibition of the BRAF-downstream MEK and ERK kinases using AZD6244/Selumetinib ${ }^{31}$ and BVD-523/ Ulixertinib $^{32}$, respectively, but not by inhibition of the upstream EGFR tyrosine kinase receptor family using AZD8931/Sapitinib ${ }^{33}$ (Fig. 2e), showing that the phenotype is due to excessive MEKERK activity. Indeed, only $24 \mathrm{~h}$ after $\mathrm{BRAF}^{\mathrm{V} 600 \mathrm{E}}$ induction, almost all direct ERK target genes ${ }^{34}$ were activated. In contrast, conditional expression of KRAS ${ }^{\mathrm{G} 12 \mathrm{~V}}$ induced RAS activity as measured by a RAS-GTP pull-down assay but had no obvious effect on bulk organoid transcription (Supplementary Fig. 1).

$\mathrm{BRAF}^{\mathrm{V} 600 \mathrm{E}}$ disrupts intestinal differentiation trajectories. To uncover potential cellular heterogeneity in response to the oncogenes, we performed single-cell transcriptome analyses. We induced FLUC control, BRAF ${ }^{\mathrm{V} 600 \mathrm{E}_{\text {- }}}$ and KRAS $\mathrm{K}^{2} \mathrm{~V}_{\text {-transgenic }}$ organoids for $24 \mathrm{~h}$, prepared single-cell suspensions, and stained them with a fluorescent antibody against the crypt cell marker CD $44^{35}$, and with a fluorescent dye to eliminate dead cells. Using single-cell sorting on the transgene-expressing organoids we next acquired samples of CD44-high crypt and CD44-low villus cells (see Supplementary Fig. 2 for FACS gating strategy), which were subjected to single-cell RNA sequencing. In total, we obtained transcriptomes of 167 cells with $>1000$ detected genes each, that were used for further analysis. Single-cell transcriptomes could be assigned to six interconnected clusters with help of k-means clustering and were visualised in a t-SNE-based representation (Fig. 3a). Mapping of signature genes for intestinal stem cells (ISCs), proliferative TA cells, differentiated enterocytes ${ }^{20}$ and secretory Paneth cells ${ }^{4}$, and the CD44 status as inferred from flow cytometry (Fig. 3b, c) confirmed the calculated differentiation trajectories (grey overlay in Fig. 3a): undifferentiated CD44-high ISC and TA cell signature genes were enriched in clusters 1 and 2 while Paneth cell marker genes were highest in cluster 2, indicating the differentiation route for secretory crypt cells; expression of enterocyte signature genes increased gradually in clusters 3-5, marking the CD44-low absorptive lineage.

We next considered the distribution of cells expressing specific transgenes (Fig. 3b): FLUC control and KRAS $\mathrm{G}^{2} \mathrm{~V}_{\text {-expressing }}$ cells intermingled throughout the clusters $1-5$ of the normal cell

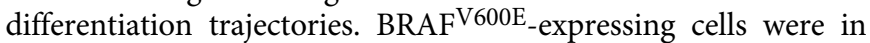
contrast depleted from the central clusters 2-4, and instead formed outsider cluster 6 , composed entirely of BRAF-induced cells. Notably, cells in cluster 6 uniformly expressed high levels of ERK target genes, regardless of whether they were sorted as CD44-high or CD44-low. Furthermore, cluster 6 cells also highly expressed Anxa10, which has been identified as a marker for BRAF-positive serrated adenoma ${ }^{36}$ (Supplementary Fig. 3). The single-cell analysis thus showed that $\mathrm{BRAF}^{\mathrm{V} 600 \mathrm{E}}$ imposed a specific gene expression programme onto intestinal cells, independent of their prior differentiation state. Transcriptomes

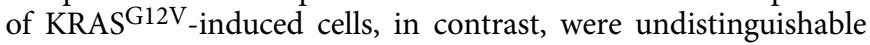

from FLUC control cells; however, we observed that KRAS G12V induced cells showed a shift towards CD44-high undifferentiated cell types compared with FLUC controls (see Supplementary Fig. 2b).

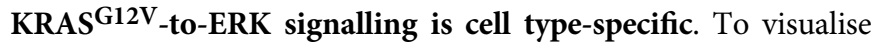
ERK activity with single-cell resolution in organoids, we employed the Fra-1-based integrative reporter of ERK (FIRE) that translates ERK kinase activity into stability of a nuclear yellowgreen venus fluorescent protein (Fig. 4a) ${ }^{23}$. FIRE fluorescence in organoids cultured in normal growth medium containing EGF was strong in crypts, whereas differentiated villus tissue was largely FIRE negative (Fig. 4b). In EGF-free medium, ERK activity in the crypt base persisted, likely due to autocrine and paracrine signals from EGF-producing Paneth cells ${ }^{4}$.

We next conditionally expressed FLUC control, $\mathrm{KRAS}^{\mathrm{G} 12 \mathrm{~V}_{-} \text {, or }}$

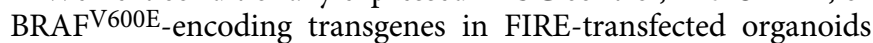
(Fig. 4c). Transgene induction was often variable, as inferred by tdTomato fluorescence, allowing to compare individual tdTomato-positive cells with transgene-negative neighbouring tissue. tdTomato-FLUC control transgene expression had no influence on FIRE activity. In contrast, expression of KRAS G12V resulted in increased FIRE signals in crypt cells, which consistently displayed stronger reporter activity compared with adjacent KRASG12V -negative cells. Surprisingly, a large majority of villus cells remained FIRE negative, despite strong tdTomatoKRASG12V positivity. We confirmed the differential signal transduction from KRASG12V to ERK using phospho-ERK immunohistochemistry (Fig. 4d). In line with our FIRE reporter data, p-ERK-positive cells were largely absent in central differentiated (Ki67-negative) villus areas of organoids, despite strong tdTomato-KRAS ${ }^{\mathrm{G} 12 \mathrm{~V}}$ staining. Taken together, our results show that ERK activity in differentiated villus epithelial cells can neither be increased by EGF in the medium nor by induction of oncogenic KRAS ${ }^{\mathrm{G} 12 \mathrm{~V}}$. However, when we induced $\mathrm{BRAF}^{\mathrm{V} 600 \mathrm{E}}$, we found widespread and strong FIRE signals across the complete organoid (Fig. 4c). This suggests a strict and cell type-specific control of signal transduction by oncogenic KRAS, but not BRAF, in intestinal epithelial cells.

Since FIRE fluorescence could distinguish cells responsive to

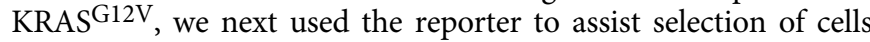
for single-cell RNA sequencing. Our aim was to define cell types with high ERK activity, either in response to KRAS ${ }^{\mathrm{G} 12 \mathrm{~V}}$ or as part of the normal cell hierarchy. For this, we induced organoids with the integrated ERK reporter for KRAS $\mathrm{G} 12 \mathrm{~V}$ or FLUC, prepared single-cell suspensions and sorted cells by FACS into 96-well plates for transcriptome analysis (see Supplementary Fig. 4 for FACS gating strategy). We focussed on single cells with high transgene (tdTomato) signal that were either positive or negative for FIRE (venus) fluorescence (Fig. 5a). In total, we obtained 197 single-cell transcriptomes. K-means clustering into eight groups and $\mathrm{t}$-SNE-based visualisation revealed the cell type distribution (Fig. 5b, c). Cluster 1 was enriched for undifferentiated crypt (ISC and TA) marker genes, whereas clusters 2-4 were defined by Paneth cell signature genes (Fig. 5d; Supplementary Fig. 5). Cluster 2 was enriched for Paneth cell markers such as $L y z 1$, encoding Lysozyme ${ }^{37}$, and several genes encoding Defensins, while other cluster-defining genes such as Mptx1 and Agr2 in cluster 4 hint at a high degree of Paneth cell heterogeneity. Clusters 5-8 formed a differentiation trajectory for absorptive cells, with Ifabp1 as the top defining gene for clusters 5-7 (Supplementary Fig. 5).

Using this information, we assessed the distribution of transcriptomes derived from KRAS $\mathrm{G}^{2} \mathrm{~V}_{\text {-induced FIRE-high cells }}$ (Fig. 5c, d). These were confined to distinct aggregates 

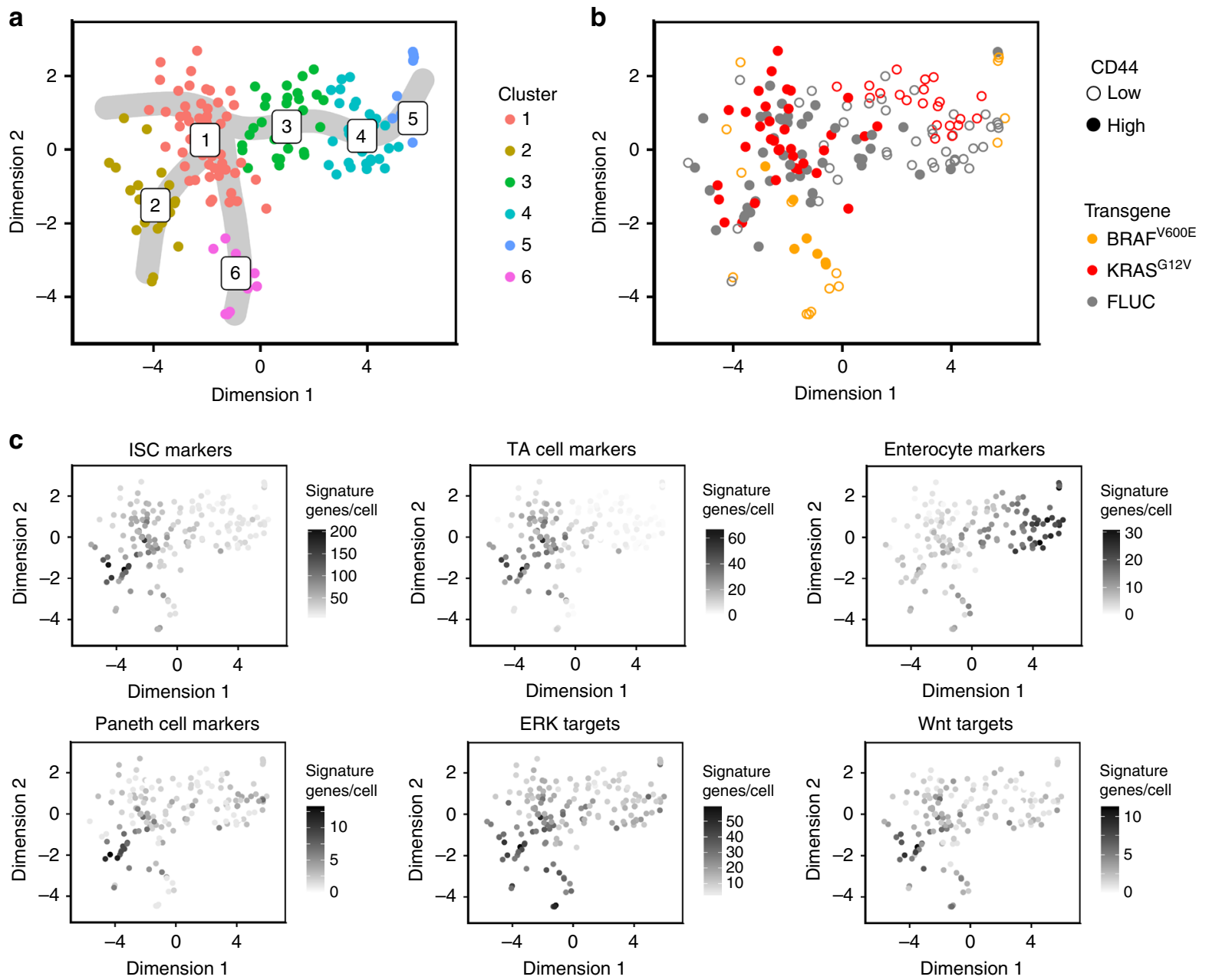

Fig. 3 Differential effects of BRAF ${ }^{V 600 E}$ or $\mathrm{KRAS}^{\mathrm{G} 12 \mathrm{~V}}$ on gene expression and intestinal cell hierarchies. All panels: t-SNE visualisations and clustering of organoid single-cell transcriptomes clustered with k-means, $24 \mathrm{~h}$ after induction of FLUC control, BRAFV600E or KRAS G12V transgenes. a Colour code for six k-means clusters, and inferred differentiation trajectories starting at cluster 1 shown as grey overlay. b Colour code for transgene and CD44 positivity, as inferred from flow cytometry. CD44 positivity was used to direct cell selection, and thus relative fractions of CD44-high and -low cells are not representative. For CD44 status of the cell populations, see Supplementary Fig. 2. c Mapping of cell- and pathway-specific differentiation signatures. Numbers of signature genes detected are given per single-cell transcriptome

encompassing the undifferentiated cell zone of cluster 1 , as well as transcriptomes inhabiting the outer right rim of the t-SNE representation that we above assigned to be derived from latestage enterocytes and Paneth cells. Immunofluorescence microscopy using the Paneth cell marker Lysozyme confirmed high FIRE activity in this cell type after $\mathrm{KRAS}^{\mathrm{G} 12 \mathrm{~V}}$ induction (Supplementary Fig. 6). In contrast, a central area of the t-SNE plot encompassing the largest clusters 5 and 6 of bulk enterocytes was almost devoid of KRAS ${ }^{\mathrm{G} 12}$-producing FIRE-high cells but harboured many KRAS ${ }^{\mathrm{G} 12 \mathrm{~V}} /$ FIRE-low cells, confirming that enterocytes generally cannot activate ERK, even when expressing oncogenic KRAS ${ }^{\mathrm{G} 12 \mathrm{~V}}$; however, a specific subset of presumably late-stage enterocytes displayed high ERK activity.

KRASG12V interacts with GSK3 $\beta$ inhibition. In order to understand how $\beta$-catenin- and MAPK-networks interact in controlling cell differentiation and ERK phosphorylation in intestinal epithelium, we performed a network perturbation study using kinase inhibitors, followed by mass cytometry in $\mathrm{KRAS}^{\mathrm{G} 12 \mathrm{~V}}$-inducible and FLUC control organoids. For this, we induced the transgenes in 3-day-old organoids, subsequently treated them with an GSK3 $\beta$ inhibitor (CHIR99021) for $24 \mathrm{~h}$ to stabilise $\beta$-catenin ${ }^{38}$, and used MEK and p38 inhibitors
(AZD6244 and LY2228820/Ralimetinib ${ }^{39}$, respectively) for $3 \mathrm{~h}$ to inhibit key kinases as part of the intestinal cell signalling network (Fig. 6a). We measured a total of 160,000 transgene-positive cells, representing 12 multiplexed samples.

To discern the immediate effects of KRASG12V and stabilised $\beta$-catenin on intestinal cell hierarchies, we assessed the distribution of cell type markers (Fig. 6b). As a positive control for the effect of GSK3 $\beta$ inhibition on $\beta$-catenin activity, treatment with CHIR99021 increased levels of the $\beta$-catenin target protein Axin $2^{40}$. We observed that both induction of KRASG12V and treatment with the GSK3 $\beta$ inhibitor, increased median levels of crypt cell markers EphB2, CD44 and CD24, and for all three proteins, KRAS ${ }^{\mathrm{G}} 12 \mathrm{~V}_{\text {-induced cells that were additionally treated }}$ with the GSK3 $\beta$ inhibitor had the highest levels. These results are in line with prior evidence that oncogenic KRAS and $\beta$-catenin activities can inhibit or reverse intestinal cell differentiation and provide clonal benefits linked to crypt cell fate $e^{41,42}$.

We used k-means clustering to allocate $\mathrm{KRAS}^{\mathrm{G} 12 \mathrm{~V}}$-induced cells to six clusters defined by levels of cell type and surface markers CD24, CD44, EphB2, Krt20 and apoptosis marker cleaved Caspase 3 (Fig. 6c). p-ERK-positive cells were enriched in clusters 5 and 6, while cleaved Caspase 3-positive cells were found in Cluster 5 (Fig. 6d, e). Based on gradual loss of the crypt cell 
a

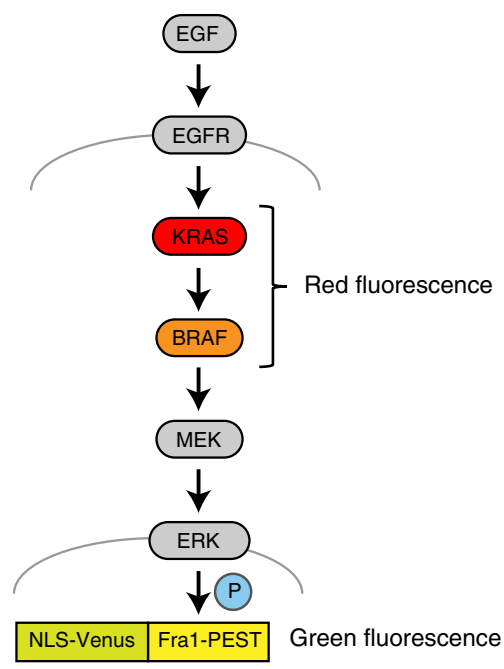

b
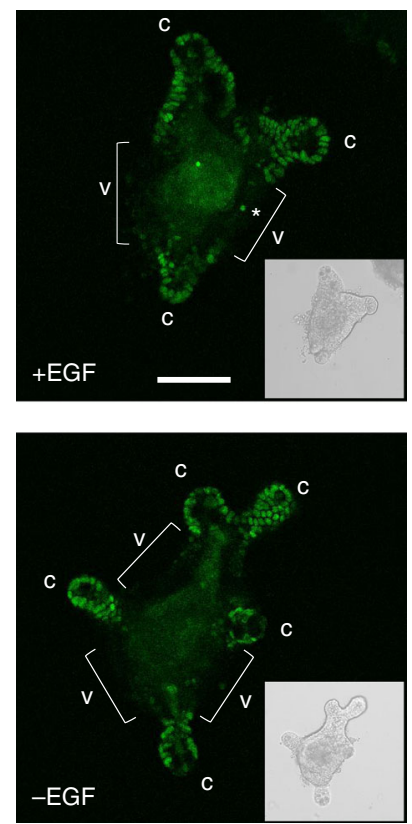

C
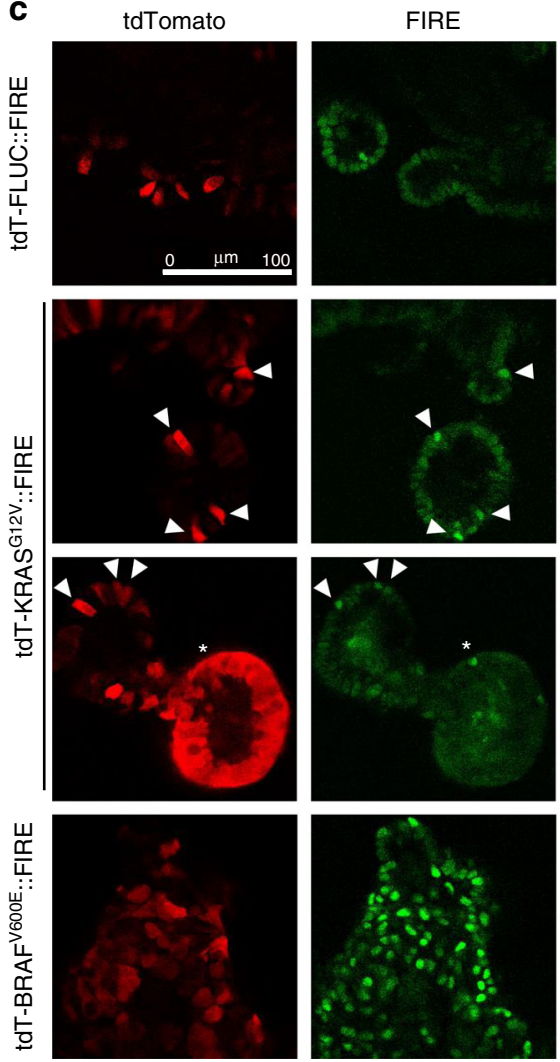

Ki67

d
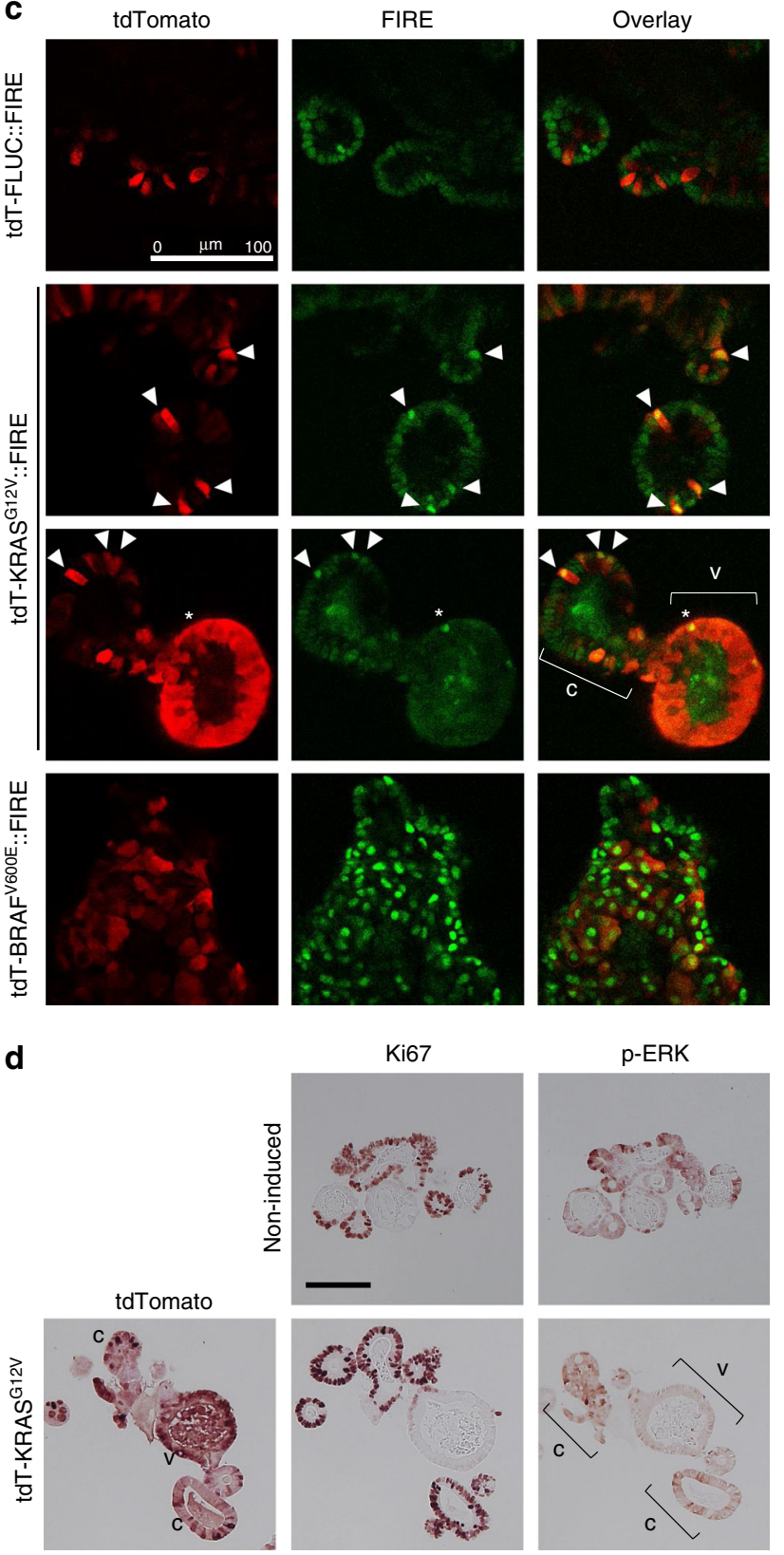

Fig. 4 Visualisation of ERK activity by FIRE reveals KRASG12V-responsive cells. a Schematic representation of signalling pathway and reporter. b FIRE activity in wild-type intestinal organoids, in the presence and absence of EGF in the culture medium, as indicated. Asterisk marks isolated FIRE-high villus cell. c Fluorescence microscopy images showing transgene expression (red), FIRE activity (green), and overlays in intestinal organoids, taken 2 days (FLUC, KRAS) or 1 day (BRAF) after transgene induction. Arrow heads mark KRASG12V/FIRE-high crypt cells, asterisk marks FIRE-high villus cell, respectively. d Immunohistochemistry of tdTomato, Ki67 and p-ERK in intestinal organoids, as indicated. In panels (c) and (d), c and v demarcate crypt and villus areas, respectively. Scale bars are $100 \mu \mathrm{m}$ in all panels

markers EphB2, CD44 and CD24, we concluded that clusters 1-4 represent a crypt-to-villus gradient, interconnecting with cluster 5 at the end of the differentiation trajectory (Fig. 6f). Clusters 3 and 4 had the lowest phospho-MEK and phospho-ERK levels, and also contained lowest levels of the $\mathrm{Wnt} / \beta$-catenin target Axin2, in agreement with differentiated villus cell status. High levels of CD24 marked p-ERK-positive cells in cluster 6 as presumptive Paneth cells ${ }^{4}$. Interestingly, we observed that fractions of cells allocated to the clusters were modified by both KRASG12V induction and GSK3 $\beta$ inhibition (Fig. 6g): both treatments increased the percentage of cells in cluster 1 , representing the presumptive undifferentiated crypt cells, as inferred from high levels of markers such as EphB2 and CD44 ${ }^{1,20}$. The combination of KRASG12V and GSK3 $\beta$ inhibition had the greatest effect and furthermore strongly decreased the fraction of cells in the apoptotic cell cluster 5 . These data suggest that oncogenic KRAS and $\beta$-catenin stabilisation can both favour crypt cell fate over differentiation, at least on the level of cell type marker expression.

Cell type-specific differences in ERK feedback regulation. To quantitatively dissect differences in signalling in the cell types, we assigned the cells measured under different perturbed conditions to the six clusters defined above according to their shortest Euclidian distance (Fig. 6c). For each condition and each cluster, we calculated average phosphorylation levels of MEK, ERK, 


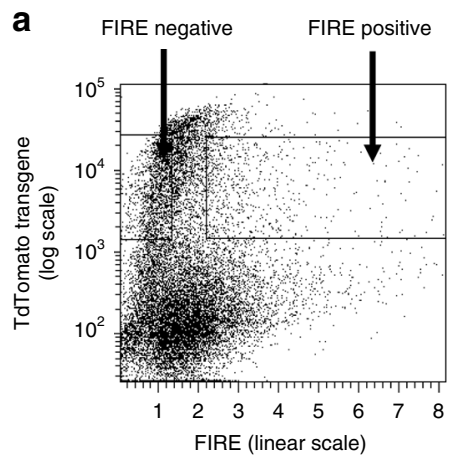

b

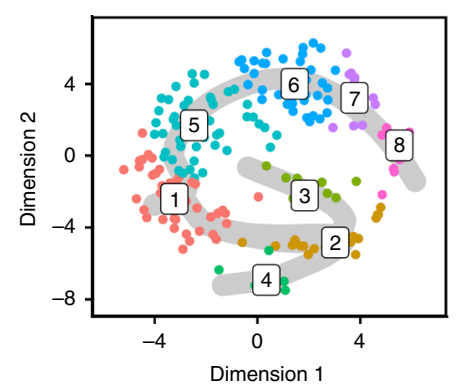

C

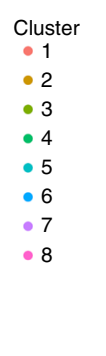

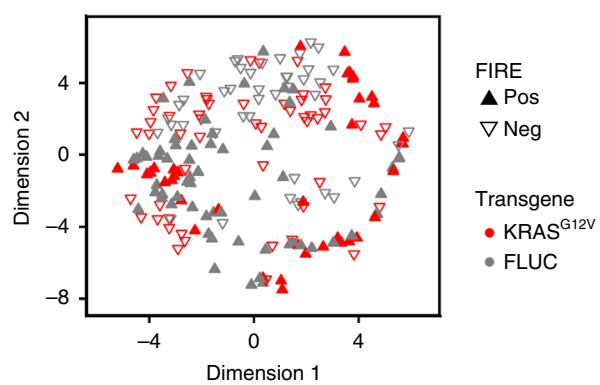

d

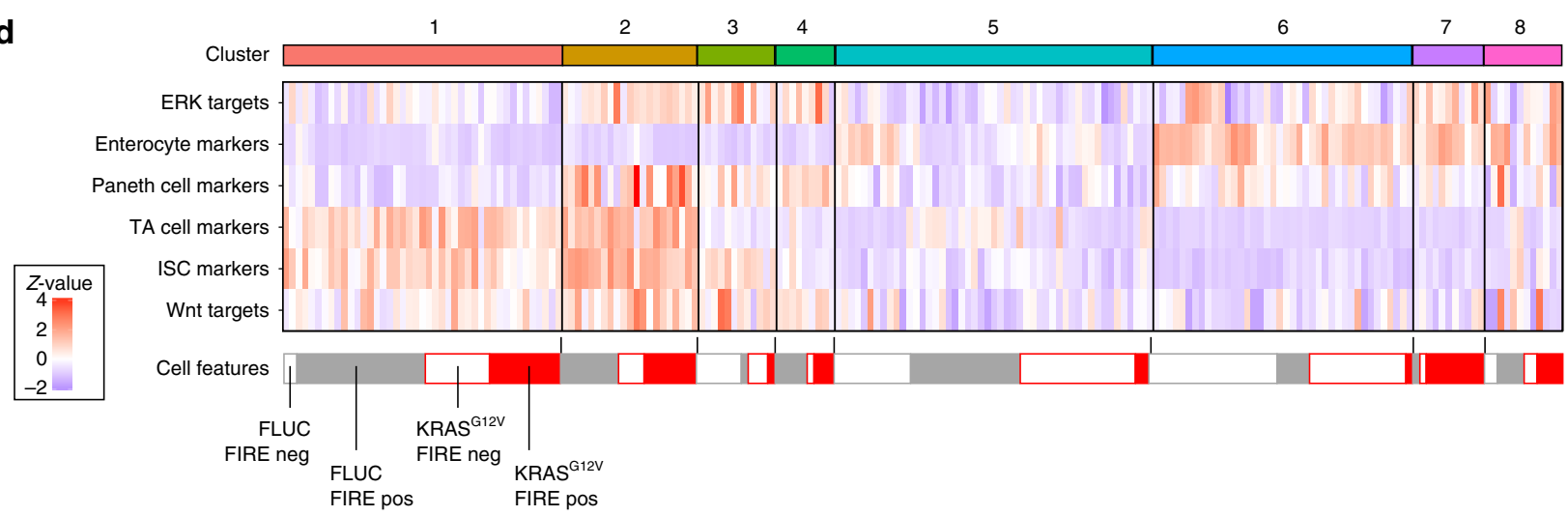

Fig. 5 Single-cell RNA sequencing reveals KRASG12V-responsive and -unresponsive organoid cells. a Fluorescence-activated cell sort gates for FIREnegative and -positive cells. $\mathbf{b}$ t-SNE visualisation colour-coded for eight clusters identified with k-means clustering. Differentiation trajectories starting at cluster 1 are shown as grey overlay. c t-SNE visualisation displaying colour codes for transgene and FIRE positivity. Filled upward-pointing triangles: FIREhigh; outlined downward-pointing triangles: FIRE-low. Red: KRASG12V; grey: FLUC. d Heatmap of z-transformed signature scores per cell for cluster cell type identification. Signature scores correspond to the number of expressed signature genes per cell normalised to gene detection rate and signature length. Blue: low target gene signature abundance; Red: high target gene signature abundance. Cluster colour codes are given above, and transgene and FIRE positivity codes are given below the heatmap

4EBP1, p38, ribosomal protein S6 and total protein levels of IKBa and Axin2, forming a Wnt-, MAPK-, NFkB- and mTOR network (Fig. 7a, b). Cellular signalling states varied strongly between the cell clusters 1 and 6 . However, comparison between FLUC control and KRAS $\mathrm{G}^{2} \mathrm{~V}$-expressing organoid cells showed that signalling within each cell type was very comparable, except for an increase in phosphorylation levels of MEK and ERK in clusters 5 and 6 in cells expressing KRAS ${ }^{G 12 V}$. This was in contrast to results from similar CyTOF experiments performed with the

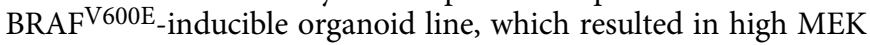
and ERK phosphorylation across all cell type clusters (Supplementary Fig. 7).

We next employed network modelling using Modular Response Analysis (MRA) ${ }^{43,44}$. This approach allows to quantify signal transmission from perturbation-response data and, by using likelihood ratio tests, to pinpoint which signalling routes are different between the clusters. The method requires fold changes in network node activity after perturbation (calculated from data shown in Fig. 7a) and a literature-derived network (as shown in Fig. 7b) as input and calculates so-called response coefficients that reflect the strength of signalling interactions. When we applied this modelling framework to the signalling perturbation data of the six clusters for the KRAS-mutant organoid data, we observed that only 5 out of 11 signalling routes significantly differed between clusters with two of them constituting the feed-forward and feedback signalling paths between MEK and ERK (Fig. 7b, c). In clusters 5 and 6, which were the clusters that showed elevated phosphorylation levels of MEK and ERK levels after KRASG12V induction, the model unveiled that these cells enable RAS-ERK signalling by two mechanisms: signal transmission from MEK to ERK was enhanced, while ERK-dependent negative feedback inhibition of RAF and upstream components was attenuated when compared with the other clusters. In contrast, clusters 3 and 4 , which exhibited the lowest phospho-ERK levels that were also not increased by KRASG12V, MEK-to-ERK feed forward signal transduction was low, and this coincided with strong ERKdependent feedback inhibition. Cluster 1, which based on surface marker expression represents undifferentiated crypt cells, had strong feedback inhibition according to the model. This cluster had intermediate phospho-ERK levels as measured by CyTOF (Fig. 6f) and was FIRE positive (Fig. 4c). However, phospho-ERK levels were unresponsive to KRAS $\mathrm{G12V}$ in this cluster and our model predicts that this could be due to strong ERK feedback.

To model differences between KRAS ${ }^{\mathrm{G} 12 \mathrm{~V}}$-induced and control cells within each cluster, we employed comparative MRA modelling that resulted in cluster-specific signalling models that consider the influence of KRAS ${ }^{\mathrm{G} 12 \mathrm{~V}}$ on the signalling network per cluster (Fig. 7d). We could discern only few differences. Most importantly, KRAS ${ }^{\mathrm{G} 12 \mathrm{~V}}$ enhanced signalling from MEK to ERK in clusters 5 and 6 . Furthermore, we observed that KRASG12V modulated the effect of Wnt/Axin2 signalling on mTOR in cluster 2 .

As our functional studies and the modelling showed that RASERK signal transduction can be differently wired between cell types, we tested whether this was also true for CRC cell lines. Indeed, when we compared ERK phosphorylation in response to transfected KRASG12V in SW48 and Caco2 CRC cells (that have 
a

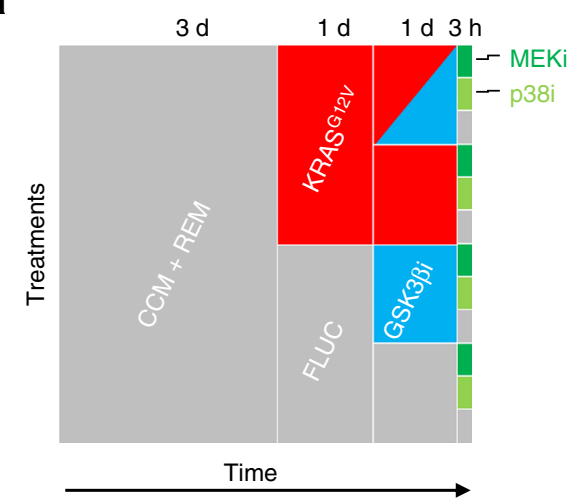

b

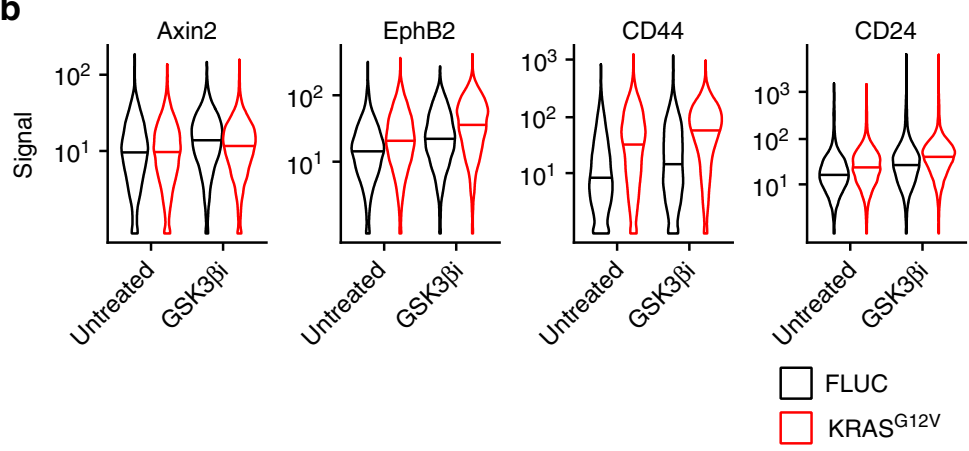

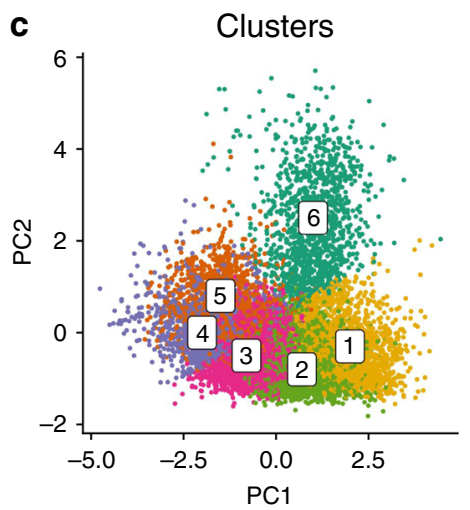

d p-ERK
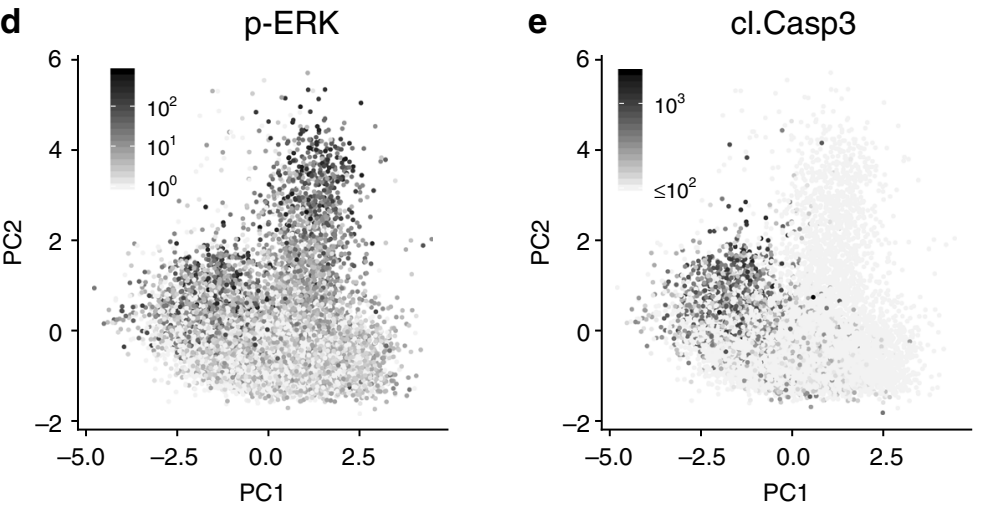

$\mathbf{f}$
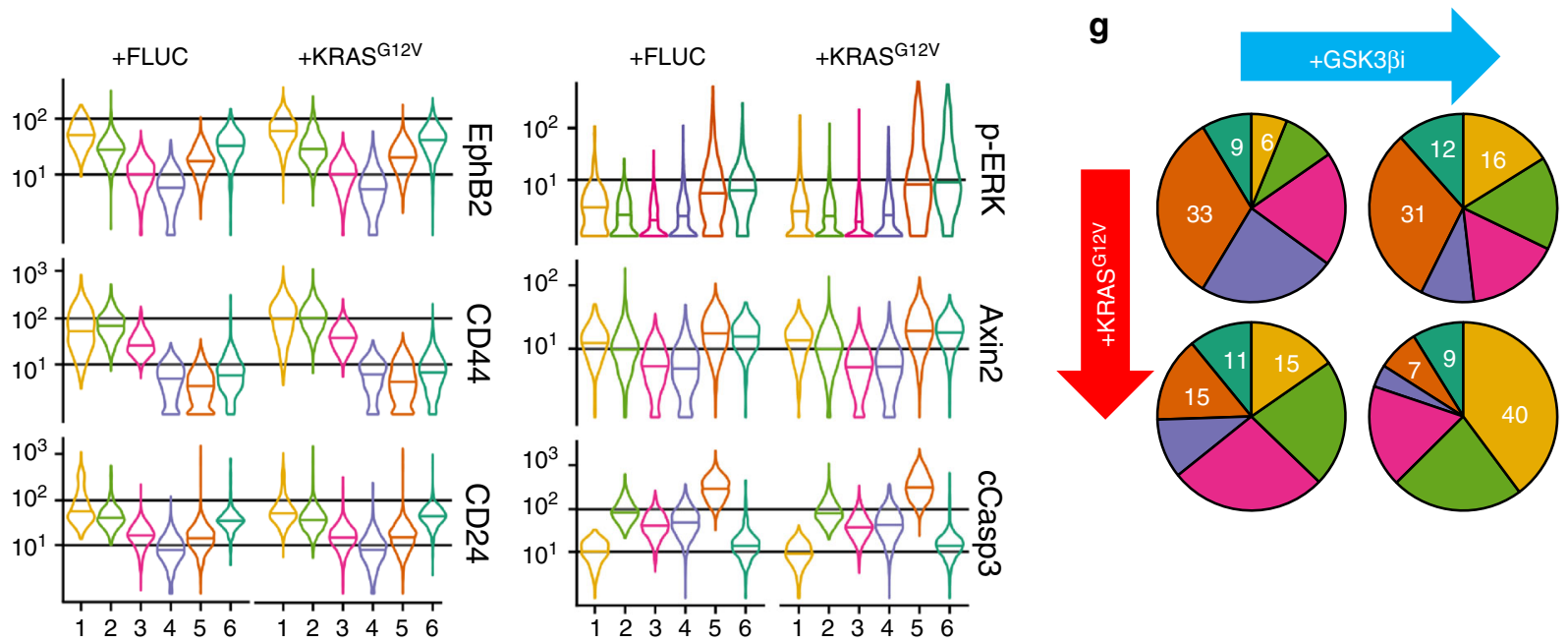

Fig. 6 CyTOF analysis reveals KRASG12V_- and GSK3 $\beta$ inhibitor-responsive p-ERK high cell clusters. a Schematics for generation of network perturbation data by CyTOF. In short, organoids were established from KRASG12V - and FLUC transgenic mice, induced for transgene expression after 3 days, and treated with GSK3 $\beta$ inhibitor for 1 day and with MEK and p38 inhibitors for $3 \mathrm{~h}$ before harvesting. Finally, 12 samples were subjected to multiplexed CyTOF analysis. b Distributions of cell type markers in organoid cells induced for FLUC or KRASG12V transgenes plus/minus GSK3 $\beta$ inhibitor treatment. Central lines of violin plots denote median values. c PCA showing colour code of k-means clustering in KRASG12V-induced cells by EphB2, CD44, CD24, Krt20 and cleaved Caspase 3 signal strength. d, e Mapping of signal strength for p-ERK and cleaved Caspase 3 on PCA, as in (c). $\mathbf{f}$ Distribution of EphB2, CD44, CD24, Axin2, p-ERK and cleaved Caspase 3 signals in clusters 1-6, as above. Central lines of violin plots denote median values. $\mathbf{g}$ Fractions of cells in clusters 1-6, in organoid cells induced for FLUC or KRASG12V transgenes plus/minus GSK3 $\beta$ inhibitor treatment. Numbers denote percentages of cells in clusters $1,5,6$. CyTOF data are available as a Source Data file

no mutations in KRAS, NRAS or BRAF), we found that Caco2 cells were $\mathrm{KRAS}^{\mathrm{G} 12 \mathrm{~V}}$-responsive, while SW48 cells were KRASG12V -insensitive, extending a recent study that shows only subtle effects of RAS mutants in SW $48^{45}$ (Supplementary Fig. 8).

Our cluster-specific signalling data (Fig. 7a) showed correlated activities of RAS-ERK, Wnt/ $\beta$-catenin and other signalling pathways, as they were generally higher in presumptive crypt cell clusters 1 and 6 , but lower in the presumptive villus enterocyte clusters 3 and 4 . Contrarily, the MRA approach suggested that Axin2 as read-out of Wnt $/ \beta$-catenin signalling was a negative regulator of RAS-ERK. We reasoned that, as we stimulated $\mathrm{Wnt} / \beta$-catenin signalling by inhibiting GSK3 $\beta$, attenuation of RAS-ERK could be caused by other targets of GSK3 $\beta$. To more directly assess the effect of $\beta$-catenin 
a
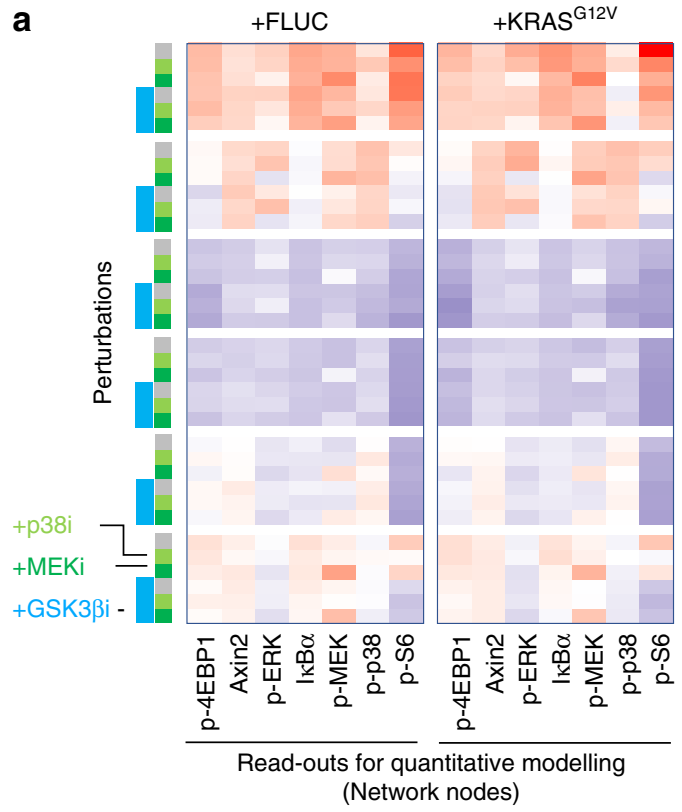

+ KRAS $^{\mathrm{G} 12 \mathrm{~V}}$

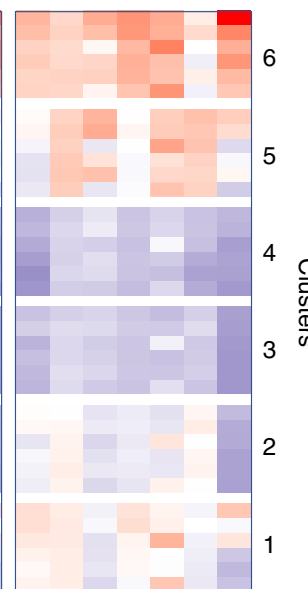

$\log 2 \mathrm{fc}$

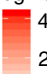

2

$-2$ b

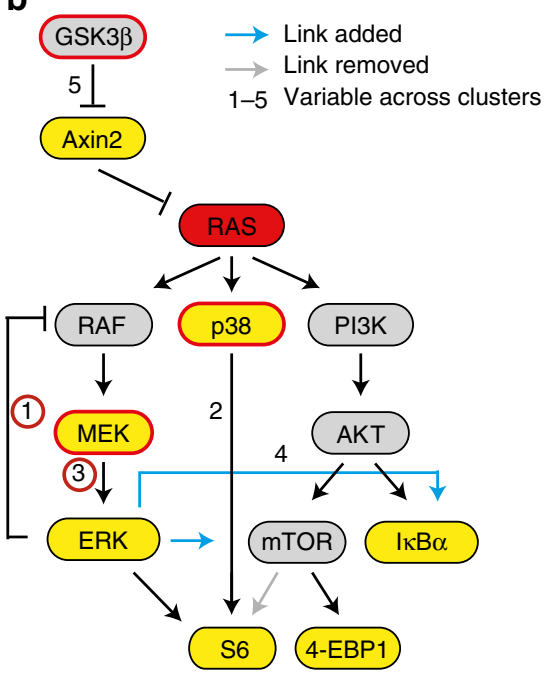

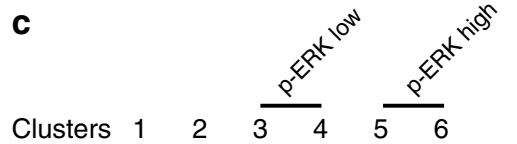

Clusters $1 \quad 2 \quad 3 \quad 4 \quad 5 \quad 6$ d

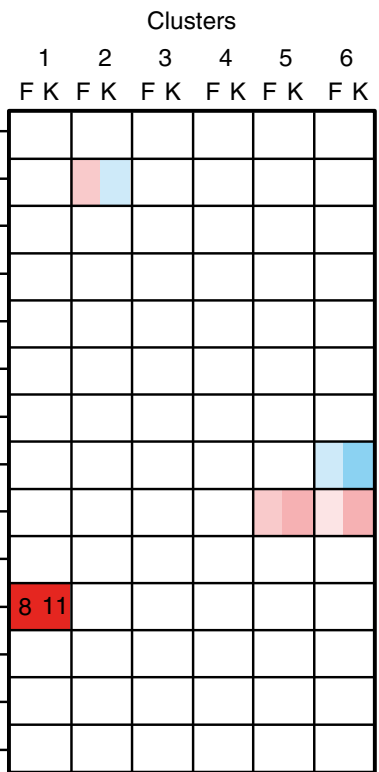

e

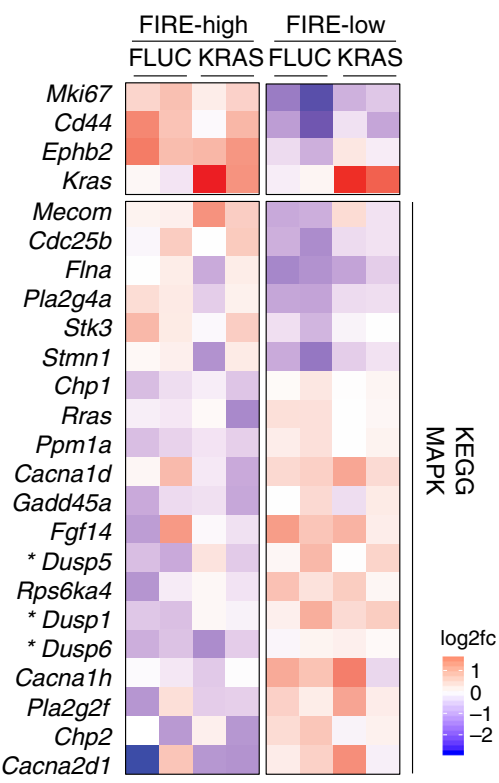

Fig. 7 Network quantification identifies cell type-specific differences in KRAS to ERK signalling. a Protein phosphorylation and abundance CyTOF data by treatment and cell clusters, as in Fig. 6c. Log2 fold changes to average untreated FLUC-induced control line are given. b Signalling network structure used for modelling. The network was re-parametrised from a starting network, using the experimental data to remove and add connections, denoted by grey and blue arrows, respectively. c Signalling quantification of identifiable network links using Modular Response Analysis. Numbers 1-5 in panels (b) and (c) show network connections with significant differences between clusters in order of detection. Red circles mark MEK-ERK and ERK-MEK connections identified as having different strengths in clusters with high vs. low ERK phosphorylation after KRASG12V induction. d MRA modelling of differences between KRASG12V induced and FLUC control cells within each cluster. K and F mark KRASG12V and FLUC control cluster pairs, respectively. Cluster pairs exhibiting KRASG12V specific differences are shown in red and blue, indicating regulation strengths. e Colour-coded gene expression data from cells sorted by high and low FIRE activity, as indicated. Upper panel shows marker genes (Mki67, encoding Ki67, for proliferative cells, Cd44 and Ephb2 for crypt cells and Kras), lower panel shows 20 significantly regulated genes between the conditions. In total, 269 genes encoding MAPK network components in KEGG were tested. Asterisks indicate dual-specificity phosphatases

on RAS-ERK, we therefore performed further experiments in transgenic organoids in which we induced transgenic stabilised $\beta$-catenin or withdrew Wnt ligands. The data show that supplementation and abrogation of $\beta$-catenin activity both result in lower ERK phosphorylation in organoids (Supplementary Fig. 9).
As the results of the modelling pinpointed differences in signal transduction from KRASG12V to ERK to feed-forward and feedback signalling between MEK and ERK, we investigated which molecular mechanism might attenuate ERK activation in intestinal cells. For this we sorted FLUC control or KRASG12V induced organoids with respect to their FIRE reporter levels, as 


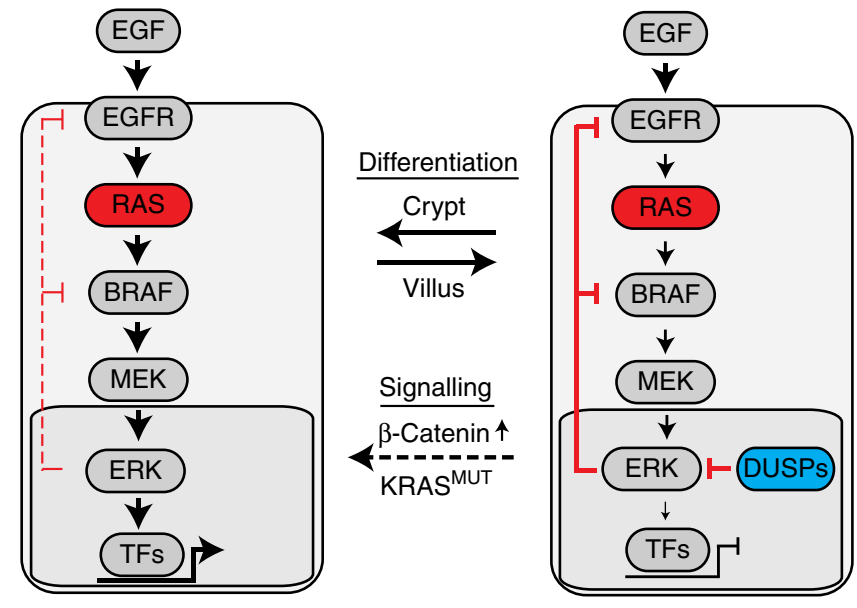

Fig. 8 Model of cell type-specific regulation of ERK activity. ERK is regulated cell type-specific and cell-intrinsic via different strengths of feedback inhibition and feed-forward signalling from MEK to ERK. Dual-specificity phosphatases (DUSPs) are important regulators of ERK activity. $\beta$-catenin and KRASG12V activities modulate cell fate decisions towards the generation of cells with high ERK activity, likely in part due to low expression of genes encoding DUSPs

above (Fig. 5a) and performed bulk low-input RNA sequencing. When inspecting 267 genes whose products are implicated in the MAPK signalling pathway, we noticed three dual-specificity phosphatases among a total of 20 differentially expressed genes (Fig. 7e). As these phosphatases are known to dephosphorylate ERK $^{46}$, we consider the DUSP1, DUSP5 and DUSP6 gene products as candidate mediators of attenuated MEK-ERK signal transmission that we observe in the differentiated cells of clusters 3-4.

\section{Discussion}

The mass cytometry, reporter assay and single-cell RNA sequencing data that we present here support a model of cell type-specific and cell-intrinsic regulation of the terminal MAP kinase ERK (Fig. 8). We found that oncogenic KRAS can activate ERK only in specific cell types of the intestinal epithelium, while other cells such as enterocytes were insensitive to ERK activation by oncogenic KRAS. Our quantitative network model suggested that ERK activity is attenuated in the latter cells by increased feedback inhibition and reduced feed-forward signalling from MEK to ERK. In agreement, transcriptome analysis showed that dual-specificity phosphatases (DUSPs) are selectively expressed in cells with low ERK activity and may therefore contribute to cell type-specific suppression of ERK. In addition, crypt cells expanded in organoids upon induction of KRASG12V, and this effect was increased when KRAS ${ }^{G 12 V}$ was combined with GSK3 $\beta$-mediated $\beta$-catenin activity.

The unexpected disparities in the levels of KRAS $\mathrm{G}^{2} \mathrm{~V}_{\text {-induced }}$ ERK phosphorylation in different cell types derived from the intestinal epithelium extend our understanding of how KRAS, the most prevalent oncogene in CRC, exerts its effects. It is of note that our study only analysed the G12V mutation of KRAS, and other mutations such as the more common G12D variant or mutations at amino acids 13, 61 or 146 may engage different effectors $^{47,48}$. Local differences of ERK activity have recently been found in clinical specimens of CRC, including KRAS-mutant $\mathrm{CRC}^{14}$. In this previous study, ERK levels were generally higher in cancer cells adjacent to stromal cells at the invasive front, and lower in more central areas of cancer specimens, in line with modulation of ERK activity by cues from the microenvironment. Our results agree with a model of dynamic ERK activity in cancer tissues, and we find in addition that ERK is not only regulated via external cues from the microenvironment, but also by the cellintrinsic differentiation state.

Quantitative modelling of ERK activity from perturbation data revealed that the markedly distinct abilities of KRASG12V to activate ERK are due to different strengths of two network connections between cell types, namely MEK to ERK feed forward and ERK to MEK negative feedback signalling. Negative feedback within the pathway is well-known, and has been linked to induction of cellular senescence ${ }^{49}$ and to therapy resistance in $\mathrm{CRC}^{44,50}$. Our study has identified dual-specificity phosphatases as candidate genes modulating MEK-to-ERK signalling during intestinal differentiation. Indeed, the Dusp1, Dusp5 and Dusp6 genes we have identified to be differentially expressed between RAS-to-ERK-responsive and -insensitive intestinal cells, encode ERK-specific phosphatases ${ }^{46}$. A protein phosphatase network, including DUSPs, has recently been found to control ERK activity and differentiation in skin ${ }^{51}$. Our study suggests the existence of similar control mechanisms in the intestine. Dual-specificity phosphatases targeting ERK have been implicated in resistance of non-small lung cancer to anti-EGFR therapy ${ }^{52}$. It remains to be seen whether differences in RAS-ERK signalling beyond the mutational status of BRAF, NRAS and KRAS have prognostic value in CRC. Today, CRC patients with wild-type status of the predictive markers KRAS, NRAS and BRAF are eligible for antiEGFR therapy ${ }^{53,54}$. However, the treatment also generates mixed outcomes among eligible patients, showing the need to identify novel markers and mechanisms contributing to differences in EGFR-RAS-ERK signal transduction and therapy response.

Paneth cells and enterocytes represent the main differentiated cell types of the intestinal epithelium, and we found that they have marked differences in their abilities to activate ERK. It is of note that the former reside in the $\mathrm{Wnt} / \beta$-catenin-high crypt compartment, while the latter inhabit a compartment with low $\beta$ catenin activity. Indeed, a functional role for $\mathrm{Wnt} / \beta$-catenin in the activation of ERK in intestinal epithelium has been proposed before $^{55}$. In our experiments using organoids, p-ERK levels were lowered when we increased or decreased $\beta$-catenin activity (Supplementary Fig. 9A, B). We hypothesise that this is due to a loss of all crypt cells or of phospho-ERK-high Paneth cells in the models with low and high $\beta$-catenin activity, respectively. Our data agree with a recent publication proposing negative interaction between $\mathrm{Wnt} / \beta$-catenin and ERK signals in intestinal stem cells $^{56}$. Indeed, activation of $\beta$-catenin by treatment with a GSK3 $\beta$ inhibitor increased the fraction of cells positive for stem cell marker CD44, but not levels of MEK or ERK phosphorylation per cell in the CyTOF experiments.

ERK activity is regulated on multiple levels, including its subcellular localisation ${ }^{57}$. We used different approaches to quantify ERK activity. While mass cytometry and capillary protein analysis measure total levels of ERK phosphorylation, the FIRE reporter assesses nuclear ERK activity only ${ }^{23}$. These activity measures for crypt cells suggest divergence of total and nuclear activity. While all crypt cells appeared FIRE positive, the presumptive undifferentiated crypt cell cluster 1 showed only intermediate levels of ERK phosphorylation in the CyTOF analysis. Further analyses will be required to understand how subcellular ERK activity is controlled in a cell type-specific manner.

In our experiments, signal transduction from BRAFV600E to ERK was relatively independent of cellular context. Extending previous studies ${ }^{29,30}$, we found that high levels of ERK activity induced by oncogenic BRAF ${ }^{\mathrm{V} 600 \mathrm{E}}$ are not tolerated in the intestine. This is in contrast to CRC and cell lines, where BRAFV600E amplifications exist and are selected for by MEK inhibition ${ }^{58,59}$. It 
thus appears that the corridor for acceptable ERK activity is tuneable during CRC progression and under selective pressure exerted, for instance, by targeted therapy. Therefore, our findings are reminiscent of the "just right" signalling model that has been proposed to explain step-wise increases of $\beta$-catenin activity in CRC progression ${ }^{60}$.

\section{Methods}

Generation of transgenic mice. Transgene cassettes were constructed by linking tdTomato to human BRAF(V600E), KRAS(G12V) and/or murine stabilised mutant Ctnnb1 (S33A, S37A, T41A, S45A) or firefly luciferase via 2A peptides, and subsequent cloning of these gene combinations into a doxycycline-inducible expression cassette flanked by heterologous loxP sites, and integrated into a previously modified Gt(ROSA)26Sor locus of F1 hybrid B6/129S6 embryonic stem cells by Cre recombinase-mediated cassette exchange ${ }^{28}$. Transgenic animal experiments were approved by Berlin authorities LAGeSo (G0185/09, G0143/14).

Organoid and cell line culture. Mouse organoid cultures were initiated and propagated as described before ${ }^{27}$, using 48 -well plates with $15 \mu$ droplets of Matrigel (Corning) per well overlaid with $300 \mu \mathrm{l}$ crypt culture medium containing EGF $(50 \mathrm{ng} / \mathrm{ml})$, Noggin $(100 \mathrm{ng} / \mathrm{ml})$ and R-Spondin1 (functionally tested from RSpondin-conditioned medium; CCM-REN). Transgenes were induced by addition of $2 \mu \mathrm{g} / \mathrm{ml}$ doxycycline to the medium.

To obtain adenomatous organoids (spheroids) after induction of stabilised $\beta$ catenin, R-Spondin was removed after induction of the transgene encoding $\beta$ catenin $^{\text {stab }}$ alone or in combination with KRAS G12V. Spheroids were dissociated with TrypLE (Gibco) for $3 \mathrm{~min}$ and Rho kinase inhibitor Y27632 (10 $\mu \mathrm{M})$ was added to the culture medium after passaging to prevent anoikis.

For viral transfection, a protocol from reference ${ }^{61}$ was employed, with modifications: organoids were cultured in the presence of Y27632 and the GSK3 $\beta$ inhibitor CHIR99021 for 2 days. Next, organoids were disaggregated into single cells using TrypLE (Gibco) for $5 \mathrm{~min}$ at $37^{\circ} \mathrm{C}$. Cell suspensions were spin-occulated in an ultra-low adhesion round bottom 96-well plate with the virus at $300 \mathrm{~g}$ for $45 \mathrm{~min}$. Subsequently, cells were resuspended in Matrigel, and cultured for 2 days in CCM-REN supplemented with Y27632 and CHIR99021. Medium was then replaced by CCM-REN containing $2 \mu \mathrm{g} / \mathrm{ml}$ puromycin to select for transfected cells. As viral transfection initially resulted in organoid pools that were heterogeneous for FIRE reporter activity, single FIRE positive organoids were manually selected and propagated before experimental analysis.

Organoid survival was scored as follows: cultures were passaged, inhibitors and doxycycline were applied with the culture medium directly after passaging. Individual wells were imaged using the $\mathrm{z}$-stack function of BioZero observation and analyser software (Keyence) on day 1 and 4 (organoids) or day 1 and 6 (spheroids), and full focus reconstructed images were used for quantification.

Patient-derived organoids (PD3Ds) were obtained from the biobank of the Charité - Universitätsmedizin Berlin and experiments were approved by the ethics commission of Charité - Universitätsmedizin Berlin (EA1/011/18). Cells were cultivated in 24-well plates (Corning) in medium containing human bFGF (20 ng/ml, Sigma Aldrich) and EGF (50 ng/ml, Sigma Aldrich), as published $^{62}$

SW48 and Caco2 CRC cells were cultured in L-15 and DMEM, respectively, supplemented with $10 \%$ foetal bovine serum. Cells were transfected using Lipofectamin 3000 Transfection Reagent (Thermo Fischer) with vectors encoding $\mathrm{BRAF}^{\mathrm{V} 600 \mathrm{E}}$, KRAS ${ }^{\mathrm{G} 12 \mathrm{~V}}$ or FLUC linked to tdTomato and the pTet-on Advanced Vector (Clontech). Cells were starved in medium containing $0.1 \%$ foetal bovine serum and induced with $2 \mu \mathrm{g} / \mathrm{ml}$ doxycycline $48 \mathrm{~h}$ after transfection. Twenty-four hours later, cells were harvested using TrypLE, washed, rested for $30 \mathrm{~min}$ at $37^{\circ} \mathrm{C}$ in starvation medium and fixed in $4 \%$ paraformaldehyde (PFA) for $15 \mathrm{~min}$ at $37^{\circ} \mathrm{C}$. Fixed cells were washed in PBS/ $1 \%$ bovine serum albumin (BSA), permeabilised in $\mathrm{MeOH}$ at $-20^{\circ} \mathrm{C}$ overnight, and immunostained with Alexa Fluor 488 mouse antiERK1/2 (pT202/pY204) antibody $(1: 10 ; 612592$, BD Bioscience) for $30 \mathrm{~min}$. Cell lines are routinely checked for mycoplasma contamination and panel-sequenced for oncogenic mutations to confirm identity.

The following inhibitors (SelleckChem) were employed: AZD6244 $(10 \mu \mathrm{M})$, BVD-523 (3 $\mu \mathrm{M})$, AZD8931 (50 nM), CHIR99021 (6 $\mu \mathrm{M})$, Gefitinib $(1 \mu \mathrm{M})$ and LY2228820 (200 nM).

Mass cytometry (CyTOF). For CyTOF analysis, we used the following preconjugated antibodies (Fluidigm) as per manufacturers recommendation: CD24 (for mouse: 150-Nd, 3150009B, for human: 169-Tm, 3169004B), CD44 (for mouse: 162-Dy, 3162030B, for human: 166-Er, 3166001B), cleaved Casp3 (142-Nd, 3142004A), p-H2AX [S139] (147-Sm, 3147016A), p-Akt [S473] (152-Sm, 3152005A), p-p38 [T180/Y182] (156-Gd, 3156002A), Ki67 (162-Dy, 3168007B), IкBa (164-Dy, 3164004A), p-ERK1/2 [T202/Y204] (171-Yb, 3171010A) and p-S6 [S235/236] (175-Lu, 3175009A).

For antibodies not available as metal-conjugates, we used the Maxpar Antibody Labeling Kit (Fluidigm) according to manufacturer's instructions for addition of the respective metal tags: Axin2 (145-Nd, Abcam, ab32197, $2 \mu \mathrm{g} / \mathrm{ml}), \mathrm{p}-\mathrm{MEK} 1 / 2$
[S217/221] (151-Eu, CST, 41G9, $2 \mu \mathrm{g} / \mathrm{ml})$, EphB2 (158-Gd, BD, 2H9, $2 \mu \mathrm{g} / \mathrm{ml}$ ), p-4e-BP1 [T37/46] (170-Er, CST, 236B4, $2 \mu \mathrm{g} / \mathrm{ml})$ and Krt20 (176-Yb, CST, D9Z1Z, $2 \mu \mathrm{g} / \mathrm{ml}$ ). The yield after conjugation was determined using a NanoDrop spectrometer measuring the absorbance at $280 \mathrm{~nm}$ wavelength.

For measurements, organoids were harvested in PBS and digested to a singlecell solution in 1:1 Accutase (Biolegend) and TrypLE (Gibco) with addition of $100 \mathrm{U} / \mathrm{ml}$ Universal Nuclease (Thermo Scientific) at $37^{\circ} \mathrm{C}$. Cells were counted and a maximum of 500,000 cells were stained with $5 \mu \mathrm{M}$ Cell-ID Cisplatin (Fluidigm) in PBS for $5 \mathrm{~min}$ at $37^{\circ} \mathrm{C}$. After washing, cells were resuspended in their respective growth medium and allowed to rest for $30 \mathrm{~min}$ at $37^{\circ} \mathrm{C}$. Subsequently, cells were resuspended in BSA/PBS solution, mixed 1:1.4 with Proteomics Stabilizer (Smart Tube Inc.) and incubated for $10 \mathrm{~min}$ at room temperature. Afterwards the cells were frozen at $-80^{\circ} \mathrm{C}$ for storage.

One day prior to analysis, cells were thawed in a $37^{\circ} \mathrm{C}$ water bath and mixed with Maxpar Cell Staining Buffer (CSB, Fluidigm). We used the Cell-ID 20-Plex Pd Barcoding Kit (Fluidigm) to label different samples and performed a downscaled version of the manufacturer's recommended protocol. Cells were washed again in CSB, then in Barcode Perm Buffer (Fluidigm). After resuspension in $200 \mu \mathrm{l}$ Barcode Perm Buffer, $25 \mu \mathrm{l}$ of the diluted Barcoding Reagents were added to the respective samples and incubated for $30 \mathrm{~min}$ at room temperature. Afterwards cells were washed twice in CSB, pooled into one tube and counted. In total, $3 \times 10^{6}$ cells were then stained with a surface antibody cocktail for $30 \mathrm{~min}$ at room temperature. After washing in CSB, cells were refixed in $2 \%$ methanol-free formaldehyde solution (Pierce; diluted in Maxpar PBS, Fluidigm) for $10 \mathrm{~min}$ at room temperature. Cells were washed in CSB and put on ice for $10 \mathrm{~min}$. Next, cells were permeabilised by adding $4{ }^{\circ} \mathrm{C}$ methanol for $15 \mathrm{~min}$. Cells were washed twice in CSB and incubated with a phospho-protein antibody cocktail for $30 \mathrm{~min}$ at room temperature. Cells were washed twice in CSB and incubated with $62.5 \mathrm{nM}$ Cell-ID Intercalator-Ir in Maxpar PBS for $20 \mathrm{~min}$ at room temperature. Cells were washed in Maxpar PBS and fixed in $2 \%$ methanol-free formaldehyde overnight at $4{ }^{\circ} \mathrm{C}$. The day after, cells were washed with CSB and then twice with Milli-Q water. Cell number was adjusted to $2.5-5 \times 10^{5} / \mathrm{ml}$ with Milli-Q water, cells were filtered through a $20 \mu \mathrm{m}$ cell strainer (CellTrics, Sysmex) and supplemented 1:10 with EQ Four Element Calibration Beads (Fluidigm). Data were acquired on a Helios CyTOF system. Mass cytometry data were normalised using the Helios software and bead-related events were removed. Doublets were excluded by filtering for DNA content ( ${ }^{191}$ Ir and ${ }^{193}$ Ir) vs. event length, and apoptotic debris removed by a filter in the platin channel $\left({ }^{195} \mathrm{Pt}\right)$. De-convolution of the barcoded sample was performed using the CATALYST R package version $1.5 .3^{63}$.

Capillary protein quantification and RAS activity assay. Protein sample pre paration and quantification of p-ERK was performed using a WES capillary system (12-230 kD Master kit $\alpha$-Rabbit-HRP; PS-MK01; Protein Simple) and the antibody p-ERK/2(T202/Y204) (1:50; \#9101, Cell Signaling). Raw p-ERK values were normalised to vinculin (1:30; \#4650; Cell Signaling). RAS activity was determined using the Ras activation Assay Biochem Kit (Cytoskeleton Inc. \#BK008), according to manufacturer's instructions and quantified using an LiCor Odyssee CXL scanner and LiCor Image Studio software.

Immunohistochemistry. Immunohistochemistry was done on PFA-fixed and paraffin-embedded tissues. Organoids were fixed in 4\% PFA for $30 \mathrm{~min}$, while intestines were fixed overnight at room temperature. Subsequently, tissues were dehydrated in a graded ethanol series, followed by xylene. Tissues were paraffineembedded, sectioned at $4 \mu \mathrm{m}$ and mounted on Superfrost Plus slides (Thermo Fisher Scientific). Sections were deparaffinised, rehydrated, bleached for $10 \mathrm{~min}$ in $3 \% \mathrm{H}_{2} \mathrm{O}_{2}$. Antigens were retrieved using $10 \mathrm{mM} \mathrm{Na}$-citrate, $\mathrm{pH} 6$ for $20 \mathrm{~min}$ at boiling temperature. The following antibodies were used: P-ERK (T202/Y204; \#4370 CellSignal); P-MEK (S217/221; \#9121 CellSignal); anti-RFP (1:200; \#600401-379 Rockland). ImmPRESS secondary antibody and NovaRED substrate kits (Vector Labs, Burlingame, CA, USA) were used for signal detection, according to manufacturer's protocols.

Immunofluorescence and microscopy. For immunofluorescence imaging, organoids were washed with PBS and fixed in-well with $4 \%$ PFA for $30 \mathrm{~min}$ at $37^{\circ} \mathrm{C}$. Fixation was stopped with PBS containing $100 \mathrm{~nm}$ Glycine. Cells were blocked and permeabilised with blocking buffer (PBS containing 1\% BSA, 0.2\% Triton X-100, $0.05 \%$ Tween-20) for at least $2.5 \mathrm{~h}$ at room temperature. Samples were incubated for $36 \mathrm{~h}$ at $4^{\circ} \mathrm{C}$ with primary antibody against lysozyme (1:250; ab108508, Abcam) diluted in blocking buffer. After washing with IF-buffer (PBS containing $0.1 \%$ BSA, $0.2 \%$ Triton X-100, 0.05\% Tween-20), samples were incubated for $24 \mathrm{~h}$ at $4{ }^{\circ} \mathrm{C}$ with secondary antibody Alexa Fluor 647 anti-rabbit (1:500, 4414, Molecular Probes) diluted in IF-buffer. Samples were counterstained for $5 \mathrm{~min}$ at room temperature using $0.5 \mu \mathrm{g} / \mathrm{ml}$ DAPI. After washing with IF-buffer, stained cultures were released from the Matrigel and collected in PBS. Samples were washed, resuspended in remaining PBS and mounted on slides using Vectashield Antifade Mounting Medium (H-1000, Vector).

Immunofluorescence and FIRE reporter images were taken with a Leica TSC SPE confocal microscope using an ACS $20 \times$ oil-immersion objective, solid-state lasers $(405,488,532$ and $635 \mathrm{~nm})$ as sources of excitation and LAS X operating 
software (Leica). Light microscopy images of cultures were taken with a Biozero microscope using a Plan Apo 4× NA 0.20 objective and Biozero observation and analyser software (Keyence)

For transmission electron microscopy, organoids were induced for $24 \mathrm{~h}$, removed from Matrigel and fixed overnight at $4{ }^{\circ} \mathrm{C}$ in Karnovsky's fixative containing 2\% PFA and 2.5\% glutaraldehyde in PBS, washed three times in PBS and embedded in $1 \%$ LM-agrose. Agorose cubes $\left(\sim 1 \mathrm{~mm}^{3}\right)$ containing several organoids were subsequently stained, using $0.5 \% \mathrm{OsO}_{4}$ in PBS ( $2.5 \mathrm{~h}$ at RT), $0.1 \%$ Tannic Acid in $100 \mathrm{mM}$ Hepes pH 7.4 ( $1 \mathrm{~h}$ at RT) and $2 \%$ Uranyle acetate $(1.5 \mathrm{~h}$ at RT), each time followed by three washing steps in PBS. Samples were dehydrated in a graded ethanol series, embedded in Spurr's resin, thin-sectioned $(70 \mathrm{~nm}$, Leica UC7) and post-contrasted as described ${ }^{64}$. Regions of $\sim 100-150 \mu \mathrm{m}^{2}$ showing representative sections through organoids were imaged on a $120 \mathrm{kV}$ Tecnai Spirit transmission electron microscope (FEI) equipped with a F416 CMOS camera (TVIPS). Micrographs were recorded automatically at a final magnification of $4400 \times(2.49 \mathrm{~nm}$ pixel size at object scale $)$ and $-10 \mu \mathrm{m}$ defocus using Leginon ${ }^{65}$ and then stitched using TrakEM2 ${ }^{66}$.

Flow cytometry and fluorescence-activated cell sorting. Flow cytometry of antip-ERK-stained CRC cells resuspended in PBS/1\% BSA cells was done using an Accuri cytometer (BD). Cells were gated for populations displaying different tdTomato fluorescence values (negative, low, medium and high), which correlates with transgene expression. For each population, the mean anti-p-ERK fluorescence values were determined and normalised to the tdTomato negative fraction of the corresponding FLUC control experiment.

For fluorescence-activated cell sorting of organoid cells, single-cell suspensions from induced organoids were prepared by digestion with TrypLE (Thermo Fisher Scientific) in the presence of $2 \mathrm{mM}$ EDTA and $200 \mathrm{U} / \mathrm{ml}$ DNAse I. Digestion was monitored by visual inspection and stopped by crypt culture medium containing $0.2 \%$ BSA. Cell suspensions were filtered through $30 \mu \mathrm{m}$ Celltrix filters and stained with an anti-CD44-antibody conjugated to Allophycocyanin (APC; clone IM7, BioLegend) and the Green or Near-IR Live/Dead Fixable Dead Cell Stain Kits (Life Tech) for subsequent exclusion of dead cells. Single cells were sorted into the 96well plates of the Precise WTA Kit with predispensed library chemistry using a BD FACSAriaII SORP (BD) and a gating strategy as displayed in Supplementary Fig. 3. Cells were sorted into quadrants of plates to minimise batch effects between plates. For later analysis of CD44 positivity of the subsets, sorts were performed as index sorts.

RNA sequencing and bioinformatic analyses. For single-cell RNA sequencing, the Precise WTA Kit (BD) was used, according to the manufacturers' instructions. Sequences were produced using NextSeq and/or HiSeq chemistry (Illumina). Cluster generation on NextSeq 500 followed the instructions of the manufacturer, at a final loading concentration of $2 \mathrm{pM}$ on a high-output-flowcell. $1 \%$ PhiX was added as quality control, at least 40 million paired reads per pool were gained during a Paired-End-75 run. Library-pools running on the HiSeq4000system were prepared according to Illumina recommendations, loaded with $200 \mathrm{pM}$ concentration and sequenced during a Paired-End-75 run. Again, $1 \% \mathrm{PhiX}$ was added as quality control, and at least 40 million read-pairs per pool were targeted.

Single-cell RNA-sequencing data were pre-processed using the BD Precise Whole Transcriptome Assay Analysis Pipeline v2.067. Quality control was performed using scater ${ }^{68}$. Read counts were normalised using the trimmed mean of median values (TMM) approach provided with edge $\mathrm{R}^{69}$. Normalised read counts were used for k-means clustering and t-SNE visualisation. Differentiation trajectories in t-SNE plots were determined using slingshot ${ }^{70}$, with intestinal stem cell cluster 1 as predefined origin. Differentially expressed genes were called on logtransformed raw counts using a hurdle model provided with R package MAST ${ }^{71}$. Top-10 signature genes per cluster were identified by comparing average gene expressions within cluster to average gene expressions across all other clusters. For bulk cell RNA sequencing, organoids were induced for $24 \mathrm{~h}$ with $2 \mu \mathrm{g} / \mathrm{ml}$ doxycycline in CCM-REN medium and subsequently dissociated using TrypLE (Thermo Fisher Scientific). RNA-seq reads were aligned to the mouse genome GRCm38 using STAR aligner with GENCODE annotation vM11. Differentially expressed genes were called using DESeq2.

Mathematical modelling. We quantified and locally adjusted the signalling networks from the KRAS-mutant perturbation data using an adjusted version of Modular Response Analysis as implemented in the R package STASNet, version $1.0 .2^{72}$ (https://github.com/molsysbio/STASNet/tree/STASNet1.0.2) as follows: As input data, we derived representative mean and standard error of the mean values from the single-cell CyTOF data (trimming the lower and upper 5\% of signals) and a literature-informed prior network. As the apparent response pattern across clusters was similar, it was decided to use a joint modelling approach, i.e. we first quantified the response coefficients for all six clusters by a single set of coefficients using a combination of latin hypercube sampling with subsequent LevenbergMarquardt fitting $\left(n=4 \times 10^{4}\right)$ and then iteratively derived and quantified the significantly different signalling coefficients between clusters using a likelihood ratio test. Afterwards we searched for biologically justifiable link extensions lacking in the network to better describe the data. The whole procedure was repeated until no further justifiable link additions could be found, followed by a removal round of statistically insignificant links.

Reporting summary. Further information on research design is available in the Nature Research Reporting Summary linked to this article.

\section{Data availability}

scRNA-seq and bulk RNA-seq data are available from GEO repository under accession numbers GSE115242 and GSE115234, respectively. Data underlying Figs. 1, 6, Supplementary Fig. 7 (CyTOF data) and Fig. 2b, d, e, and Supplementary Figs. 1c, 6, 8, 9 are provided as source data files. Modelling information is available as a source data file.

Received: 6 June 2018 Accepted: 12 June 2019

Published online: 02 July 2019

\section{References}

1. Beumer, J. \& Clevers, H. Regulation and plasticity of intestinal stem cells during homeostasis and regeneration. Development 143, 3639-3649 (2016).

2. Stelniec-Klotz, I. et al. Reverse engineering a hierarchical regulatory network downstream of oncogenic KRAS. Mol. Syst. Biol. 8, 601 (2012).

3. Sasaki, N. et al. Reg4+deep crypt secretory cells function as epithelial niche for Lgr5+stem cells in colon. Proc. Natl Acad. Sci. USA 113, E5399-E5407 (2016)

4. Sato, T. et al. Paneth cells constitute the niche for Lgr5 stem cells in intestinal crypts. Nature 469, 415-418 (2010)

5. Pylayeva-Gupta, Y., Grabocka, E. \& Bar-Sagi, D. RAS oncogenes: weaving a tumorigenic web. Nat. Rev. Cancer 11, 761-774 (2011).

6. Holderfield, M., Deuker, M. M., McCormick, F. \& McMahon, M. Targeting RAF kinases for cancer therapy: BRAF-mutated melanoma and beyond. Nat. Rev. Cancer 14, 455-467 (2014).

7. Ciardiello, F. et al. Differential expression of epidermal growth factor-related proteins in human colorectal tumors. Proc. Natl Acad. Sci. USA 88, 7792-7796 (1991)

8. Papke, B. \& Der, C. J. Drugging RAS: know the enemy. Science 355, 1158-1163 (2017).

9. Fearon, E. R. \& Vogelstein, B. A genetic model for colorectal tumorigenesis. Cell 61, 759-767 (1990).

10. Fearon, E. R. Molecular genetics of colorectal cancer. Annu. Rev. Pathol. 6, 479-507 (2011)

11. Rad, R. et al. A genetic progression model of $\mathrm{Braf}(\mathrm{V} 600 \mathrm{E})$-induced intestinal tumorigenesis reveals targets for therapeutic intervention. Cancer Cell 24, 15-29 (2013)

12. Yachida, S., Mudali, S., Martin, S. A., Montgomery, E. A. \& IacobuzioDonahue, C. A. Beta-catenin nuclear labeling is a common feature of sessile serrated adenomas and correlates with early neoplastic progression after BRAF activation. Am. J. Surg. Pathol. 33, 1823-1832 (2009).

13. Morkel, M., Riemer, P., Sers, C., Bläker, H. \& Sers, C. Similar but different: distinct roles for KRAS and BRAF oncogenes in colorectal cancer development and therapy resistance. Oncotarget 6, 20785-20800 (2015)

14. Blaj, C. et al. Oncogenic effects of high MAPK activity in colorectal cancer mark progenitor cells and persist irrespective of RAS mutations. Cancer Res. 77, 1763-1774 (2017).

15. Hlubek, F. et al. Heterogeneous expression of Wnt/beta-catenin target genes within colorectal cancer. Int. J. Cancer 121, 1941-1948 (2007).

16. Snippert, H. J. et al. Prominin-1/CD133 marks stem cells and early progenitors in mouse small intestine. Gastroenterology 136, 2187-2194.e1 (2009).

17. Zhu, L. et al. Prominin 1 marks intestinal stem cells that are susceptible to neoplastic transformation. Nature 457, 603-607 (2009).

18. O’Brien, C. A., Pollett, A., Gallinger, S. \& Dick, J. E. A human colon cancer cell capable of initiating tumour growth in immunodeficient mice. Nature $\mathbf{4 4 5}$ 106-110 (2007).

19. Todaro, M. et al. Colon cancer stem cells dictate tumor growth and resist cell death by production of interleukin-4. Cell Stem Cell 1, 389-402 (2007).

20. Merlos-Suárez, A. et al. The intestinal stem cell signature identifies colorectal cancer stem cells and predicts disease relapse. Cell Stem Cell 8, 511-524 (2011).

21. Sadanandam, A. et al. A colorectal cancer classification system that associates cellular phenotype and responses to therapy. Nat. Med. 19, 619-625 (2013).

22. Kreso, A. et al. Variable clonal repopulation dynamics influence chemotherapy response in colorectal cancer. Science 339, 543-548 (2013).

23. Albeck, J. G., Mills, G. B. \& Brugge, J. S. Frequency-modulated pulses of ERK activity transmit quantitative proliferation signals. Mol. Cell 49, 249-261 (2013). 
24. Grün, D. et al. Single-cell messenger RNA sequencing reveals rare intestinal cell types. Nature 525, 251-255 (2015).

25. Li, H. et al. Reference component analysis of single-cell transcriptomes elucidates cellular heterogeneity in human colorectal tumors. Nat. Genet. 49, 708-718 (2017).

26. Bodenmiller, B. et al. Multiplexed mass cytometry profiling of cellular states perturbed by small-molecule regulators. Nat. Biotechnol. 30, 858-867 (2012).

27. Sato, T. et al. Single Lgr5 stem cells build crypt-villus structures in vitro without a mesenchymal niche. Nature 459, 262-265 (2009).

28. Vidigal, J. A. et al. An inducible RNA interference system for the functional dissection of mouse embryogenesis. Nucleic Acids Res. 38, e122 (2010).

29. Riemer, P. et al. Transgenic expression of oncogenic BRAF induces loss of stem cells in the mouse intestine, which is antagonized by $\beta$-catenin activity. Oncogene 34, 3164-3175 (2015).

30. Tong, K. et al. Degree of tissue differentiation dictates susceptibility to BRAFdriven colorectal cancer. Cell Rep. 21, 3833-3845 (2017).

31. Yeh, T. C. et al. Biological characterization of ARRY-142886 (AZD6244), a potent, highly selective mitogen-activated protein kinase kinase $1 / 2$ inhibitor. Clin. Cancer Res. 13, 1576-1583 (2007).

32. Sullivan, R. J. et al. First-in-class ERK1/2 inhibitor ulixertinib (BVD-523) in patients with MAPK mutant advanced solid tumors: Results of a phase I doseescalation and expansion study. Cancer Discov. 8, 184-195 (2018).

33. Barlaam, B. et al. Discovery of AZD8931, an equipotent, reversible inhibitor of signaling by EGFR, HER2, and HER3 receptors. ACS Med. Chem. Lett. 4, 742-746 (2013).

34. Uhlitz, F. et al. An immediate-late gene expression module decodes ERK signal duration. Mol. Syst. Biol. 13, 928 (2017).

35. Wang, F. et al. Isolation and characterization of intestinal stem cells based on surface marker combinations and colony-formation assay. Gastroenterology 145, 383-95.e1-21 (2013).

36. Gonzalo, D. H. et al. Gene expression profiling of serrated polyps identifies annexin $\mathrm{A} 10$ as a marker of a sessile serrated adenoma/polyp. J. Pathol. 230, 420-429 (2013).

37. Peeters, T. \& Vantrappen, G. The Paneth cell: a source of intestinal lysozyme. Gut 16, 553-558 (1975).

38. Yin, X. et al. Niche-independent high-purity cultures of Lgr5+ intestinal stem cells and their progeny. Nat. Methods 11, 106-112 (2014).

39. Ishitsuka, K. et al. p38 mitogen-activated protein kinase inhibitor LY2228820 enhances bortezomib-induced cytotoxicity and inhibits osteoclastogenesis in multiple myeloma; therapeutic implications. Br. J. Haematol. https://doi.org/ 10.1111/j.1365-2141.2008.07044.x (2008).

40. Lustig, B. et al. Negative feedback loop of Wnt signaling through upregulation of conductin/axin2 in colorectal and liver tumors. Mol. Cell. Biol. 22, 1184-1193 (2002).

41. Schwitalla, S. et al. Intestinal tumorigenesis initiated by dedifferentiation and acquisition of stem-cell-like properties. Cell 152, 25-38 (2013).

42. Vermeulen, L. et al. Defining stem cell dynamics in models of intestinal tumor initiation. Science 342, 995-998 (2013).

43. Kholodenko, B. N. et al. Untangling the wires: a strategy to trace functional interactions in signaling and gene networks. Proc. Natl Acad. Sci. USA https:// doi.org/10.1073/pnas.192442699 (2002).

44. Klinger, B. et al. Network quantification of EGFR signaling unveils potential for targeted combination therapy. Mol. Syst. Biol. 9, 673 (2013).

45. Hood, F. E. et al. Isoform-specific Ras signaling is growth factor dependent. Mol. Biol. Cell mbc-E18 (2019).

46. Kidger, A. M. \& Keyse, S. M. The regulation of oncogenic Ras/ERK signalling by dual-specificity mitogen activated protein kinase phosphatases (MKPs). Semin. Cell Dev. Biol. https://doi.org/10.1016/j.semcdb.2016.01.009 (2016).

47. Hunter, J. C. et al. Biochemical and Structural Analysis of Common CancerAssociated KRAS Mutations. Mol. Cancer Res. https://doi.org/10.1016/j. semcdb.2016.01.009 (2015)

48. Hammond, D. E. et al. Differential reprogramming of isogenic colorectal cancer cells by distinct activating KRAS mutations. J. Proteome Res. https:// doi.org/10.1021/pr501191a (2015).

49. Courtois-Cox, S. et al. A negative feedback signaling network underlies oncogene-induced senescence. Cancer Cell https://doi.org/10.1016/j. ccr.2006.10.003 (2006).

50. Prahallad, A. et al. Unresponsiveness of colon cancer to $B R A F(V 600 \mathrm{E})$ inhibition through feedback activation of EGFR. Nature 483, 100-103 (2012).

51. Mishra, A. et al. A protein phosphatase network controls the temporal and spatial dynamics of differentiation commitment in human epidermis. Elife https://doi.org/10.7554/eLife.27356 (2017).

52. Phuchareon, J., McCormick, F., Eisele, D. W. \& Tetsu, O. EGFR inhibition evokes innate drug resistance in lung cancer cells by preventing Akt activity and thus inactivating Ets-1 function. Proc. Natl Acad. Sci. USA https://doi.org/ 10.1073/pnas.1510733112 (2015).
53. Karapetis, C. S. et al. K-ras mutations and benefit from cetuximab in advanced colorectal cancer. N. Engl. J. Med. 359, 1757-1765 (2008).

54. Amado, R. G. et al. Wild-type KRAS is required for panitumumab efficacy in patients with metastatic colorectal cancer. J. Clin. Oncol. 26, 1626-1634 (2008).

55. Phelps, R. A. et al. A two-step model for colon adenoma initiation and progression caused by APC loss. Cell 137, 623-634 (2009).

56. Kabiri, Z. et al. Wnt signaling suppresses MAPK-driven proliferation of intestinal stem cells. J. Clin. Invest. https://doi.org/10.1172/JCI99325 (2018).

57. Lenormand, P., Brondello, J. M., Brunet, A. \& Pouysségur, J. Growth factorinduced p42/p44 MAPK nuclear translocation and retention requires both MAPK activation and neosynthesis of nuclear anchoring proteins. J. Cell Biol. https://doi.org/10.1083/jcb.142.3.625 (1998).

58. Corcoran, R. B. et al. BRAF gene amplification can promote acquired resistance to MEK inhibitors in cancer cells harboring the BRAF V600E mutation. Sci. Signal. 3, ra84 (2010).

59. Little, A. S. et al. Amplification of the driving oncogene, KRAS or BRAF, underpins acquired resistance to MEK1/2 inhibitors in colorectal cancer cells. Sci. Signal. 4, ra17 (2011).

60. Albuquerque, C. et al. The 'just-right' signaling model: APC somatic mutations are selected based on a specific level of activation of the betacatenin signaling cascade. Hum. Mol. Genet. 11, 1549-1560 (2002).

61. Fujii, M., Matano, M., Nanki, K. \& Sato, T. Efficient genetic engineering of human intestinal organoids using electroporation. Nat. Protoc. 10, 1474-1485 (2015).

62. Schütte, M. et al. Molecular dissection of colorectal cancer in pre-clinical models identifies biomarkers predicting sensitivity to EGFR inhibitors. Nat. Commun. 8, 14262 (2017).

63. Chevrier, S. et al. Compensation of signal spillover in suspension and imaging mass cytometry. Cell Syst. https://doi.org/10.1016/j.cels.2018.02.010 (2018).

64. Matz, P. et al. Footprint-free human fetal foreskin derived iPSCs: A tool for modeling hepatogenesis associated gene regulatory networks. Sci. Rep. https:// doi.org/10.1038/s41598-017-06546-9 (2017)

65. Suloway, C. et al. Automated molecular microscopy: the new Leginon system. J. Struct. Biol. 151, 41-60 (2005).

66. Cardona, A. et al. TrakEM2 software for neural circuit reconstruction. PLoS ONE 7, e38011 (2012).

67. Fu, G. K., Wilhelmy, J., Stern, D., Fan, H. C. \& Fodor, S. P. A. Digital encoding of cellular mRNAs enabling precise and absolute gene expression measurement by single-molecule counting. Anal. Chem. 86, 2867-2870 (2014).

68. McCarthy, D. J., Campbell, K. R., Lun, A. T. L. \& Wills, Q. F. Scater: preprocessing, quality control, normalization and visualization of single-cell RNA-seq data in R. Bioinformatics 33, 1179-1186 (2017).

69. Robinson, M. D., McCarthy, D. J. \& Smyth, G. K. edgeR: a Bioconductor package for differential expression analysis of digital gene expression data. Bioinformatics 26, 139-140 (2010).

70. Street, K. et al. Slingshot: Cell lineage and pseudotime inference for single-cell transcriptomics. bioRxiv 128843, https://doi.org/10.1101/128843 (2017).

71. Finak, G. et al. MAST: a flexible statistical framework for assessing transcriptional changes and characterizing heterogeneity in single-cell RNA sequencing data. Genome Biol. 16, 278 (2015).

72. Dorel, M. et al. Modelling signalling networks from perturbation data Bioinformatics https://doi.org/10.1093/bioinformatics/bty473 (2018).

\section{Acknowledgements}

The authors gratefully acknowledge excellent technical assistance by Anja Sieber (IRI Life Sciences and Charité Universitätsmedizin Berlin), Gaby Bläss and Sonja Banko (MPIMG Berlin) for RAS activity assays, mouse genotyping and mouse care, respectively. The authors also gratefully acknowledge the help of lab students Ekaterina Eroshok and Maximilian Anders (Molecular Medicine Masters programme, Charité Universitätsmedizin Berlin) with immunohistochemistry in the early phase of this project. We received the FIRE plasmid as a kind gift from John Albeck, UC Davis. The work was in part funded by Deutsche Forschungsgemeinschaft (MO2783/2-1 to M.M.), Berlin School of Integrative Oncology (to N.B. and M.M.), the German Cancer Consortium DKTK (to N.B. and M.M.), the Federal Ministry of Education and Research BMBF (StemNet 01EK1604B to N.B.; ColoSys 031L0081 to C.S. and N.B.) and the Berlin Institute of Health (to N.B., C.S. and M.M.)

\section{Author contributions}

R.B., T.S., M.L., P.R., C.G.-T., S.S., D.K., N.M., B.F. and I.A.E.-S. conducted, analysed and interpreted experiments; F.U., T.S. and B.K. performed bioinformatic analyses; M.M., N.B., C.S., P.R., T.M. and B.G.H. conceived, designed, interpreted experiments and/or supervised parts of the study; M.M., N.B. and B.K. wrote the paper. 


\section{Additional information}

Supplementary Information accompanies this paper at https://doi.org/10.1038/s41467019-10954-y.

Competing interests: The authors declare no competing interests.

Reprints and permission information is available online at http://npg.nature.com/ reprintsandpermissions/

Peer review information: Nature Communications thanks Rony Seger and other anonymous reviewer(s) for their contribution to the peer review of this work. Peer reviewer reports are available.

Publisher's note: Springer Nature remains neutral with regard to jurisdictional claims in published maps and institutional affiliations. (c) (i) Open Access This article is licensed under a Creative Commons Attribution 4.0 International License, which permits use, sharing, adaptation, distribution and reproduction in any medium or format, as long as you give appropriate credit to the original author(s) and the source, provide a link to the Creative Commons license, and indicate if changes were made. The images or other third party material in this article are included in the article's Creative Commons license, unless indicated otherwise in a credit line to the material. If material is not included in the article's Creative Commons license and your intended use is not permitted by statutory regulation or exceeds the permitted use, you will need to obtain permission directly from the copyright holder. To view a copy of this license, visit http://creativecommons.org/ licenses/by/4.0/.

(C) The Author(s) 2019 